\title{
Direct calculation of critical points in parameter sensitive systems
}

\author{
Behrang Moghaddasie ${ }^{\mathrm{a}}$, Ilinca Stanciulescu ${ }^{\mathrm{b}, *}$ \\ ${ }^{a}$ Department of Civil Engineering, Ferdowsi University of Mashhad, P.O. Box 91775-1111, \\ Mashhad, Iran \\ ${ }^{b}$ Department of Civil and Environmental Engineering, Rice University, Houston TX \\ 77005-1892, U.S.A.
}

\begin{abstract}
At critical points along the equilibrium path, sudden and sometimes catastrophic changes in the structural behaviour are observed. The equilibrium path, load-bearing capacity and locations of critical points can be sensitive to variations in parameters, such as geometrical imperfections, multi-parameter loadings, temperature and material properties. This paper introduces an incrementaliterative procedure to directly calculate the critical load for parameterized elastic structures. A modified Newton's method is proposed to simultaneously set the residual force and the minimum eigenvalue of the tangent stiffness matrix to zero by using an iterative algorithm. To demonstrate the performance of this method, numerical examples are presented.
\end{abstract}

Keywords: Stability boundary, Sensitivity analysis, Control parameter, Critical load, Arc-length method, Conservative systems

\section{Introduction}

Non-linear structural systems are usually sensitive to variations in geometrical, mechanical and loading parameters. In many non-linear structural systems, variations in the control parameters significantly affect the equilibrium path and, subsequently, the buckling strength. Columns, trusses, shallow arches and

\footnotetext{
*Corresponding author: Ilinca Stanciulescu, Department of Civil and Environmental Engineering, Rice University, Houston TX 77005-1892, U.S.A. E-mail: ilinca.s@rice.edu
}

Preprint submitted to Computers and Structures

October 11, 2012 
thin-walled structures are examples of such systems [1, 2]. Consequently, a precise relationship between the load-bearing capacity of structures and these control parameters is required for robust analysis and design. In mechanicalstructural problems, it can be assumed that the magnitude of imperfections changes through one or more independent parameters [3]. As a result, the equilibrium equations depend on these parameters, and, so does the critical load.

This paper introduces a numerical procedure to find the influence of control parameters on critical points. Since these points play an important role in the post-buckling behaviour of structures, their detection is needed for choosing a suitable numerical strategy while tracing the equilibrium path. Techniques for tracing the equilibrium path were extensively discussed in the literature $[4,5,6]$. A broad class of numerical methods is utilized, many based on Newton's approach. In these methods, a number of discrete equilibrium points are obtained through an incremental-iterative procedure $[7,8,9]$. Some of these techniques select a wrong path or become divergent when they reach critical points (e.g., limit, simple bifurcation and multi-bifurcation points). Many efforts have been made by researchers in detecting critical points $[10,11,12]$. At these points, the tangent stiffness matrix becomes singular. Therefore, most of the proposed methods use this characteristic to detect critical points by adding the singularity constraint (in terms of the properties of the stiffness matrix) to the governing equations of the system, and applying an iterative procedure for the solution $[13,14,15]$.

Initial geometric defects, load imperfections and thermal stresses are examples of imperfections that can influence the load-bearing capacity of a structure $[16,17]$. The sensitivity analysis of critical states has been investigated often $[1,18]$, mostly by simultaneously perturbing the equilibrium equation and the singularity constraint in the vicinity of the critical point $[19,20,21]$. This method is compatible with the finite element method. The drawback is that it requires the calculation of higher order derivatives of the total energy function. Moreover, its range of validity is restricted to the neighbourhood of the critical point. Another technique with similar advantages and disadvantages, the 
Lyapunov-Schmidt-Koiter asymptotic approach, is based on regularizing the governing equations by a perturbation parameter $[22,23,24]$. More recently, researchers have introduced incremental-iterative numerical methods that directly calculate the buckling strength of parameterized (imperfect) structures $[25,26]$. The superiority of these techniques in comparison with perturbation methods consists in that the equilibrium equations and the singularity constraint are simultaneously enforced via an iterative procedure. Consequently, errors will not increase for larger variations in parameters.

In this paper, a modified Newton's method is proposed to solve the equilibrium equations and the singularity constraint through an incremental-iterative procedure. The method simultaneously sets the residual force and the minimum eigenvalue to zero. Since the locus of critical points in non-conservative systems is dependent on the traced equilibrium path, and the proposed method directly calculates a set of critical points without tracking relative equilibrium paths, only conservative systems (e.g., elastic structures) are considered in this paper. The superiority of this method in comparison with previous works consists in: (a) changes in the buckling mode do not lead to divergence; (b) errors will not increase when the magnitude of control parameters grows; (c) the technique has the capability to track limit, simple bifurcation and multi-bifurcation points in the entire parameter space; (d) since the proposed method is an incrementaliterative procedure, and each critical point is directly computed from the previous one, it can be applied for structures with large pre-critical displacements without using globalization techniques (which would be necessary for the computation of the singular point from the unloaded state). The ability to use it in conjunction with the finite element method is also an advantage of the approach we introduce.

A brief outline of this paper is as follows: In Section 2, some basic equations are given and the spherical arc-length method is briefly described. The characteristics of critical points are investigated. A direct strategy for calculating the critical load is presented. Section 3 reviews the governing equations of parameterized systems. The formulation and numerical implementation of proposed method for parameter sensitivity analysis of critical points are introduced in 
Section 4. Numerical examples in Section 5 show the robustness and accuracy of the proposed approach in tracing critical points for various types of control parameters and imperfections. Finally, concluding remarks are given in Section 6.

\section{Equilibrium path}

In perfect elastic structures, the total potential energy $\Pi$ is a function of the nodal displacements vector $\mathbf{u} \in \mathbb{R}^{n}$ and of the load factor $p \in \mathbb{R}$. This energy can be expressed as the summation of the internal strain energy $\Phi$ and the external work done by the applied load(s). For structures under a displacement independent loading, $\Pi$ is

$$
\Pi(\mathbf{u}, p)=\Phi(\mathbf{u})-p \mathbf{q}^{T} \mathbf{u}
$$

where $\mathbf{q}$ represents the reference load vector and the superscript $T$ denotes the transpose of the vector or matrix. The first derivative of the total potential energy with respect to $\mathbf{u}$ gives the residual force $\mathbf{r}$, which contains out-of-balance forces. Consequently, the equilibrium equation is as follows:

$$
\mathbf{r}(\mathbf{u}, p)=\mathbf{F}_{\text {int }}(\mathbf{u})-p \mathbf{q}=\mathbf{0} .
$$

Here, $\mathbf{F}_{\text {int }}=\partial \Phi / \partial \mathbf{u}$ denotes the internal force calculated by the first derivative of the strain energy with respect to the nodal displacement vector and $p \mathbf{q}$ is the external load. The set of points that satisfy Eq. (2) is called equilibrium path.

There exist many numerical techniques to follow the equilibrium path $[5,27$, 28]. Most of them are based on incremental-iterative strategies. First, new increments of load and nodal displacement (denoted by $\Delta p$ and $\Delta \mathbf{u}$, respectively) are added to the previous equilibrium state. The increment can be obtained from

$$
\mathbf{K}_{T}(\mathbf{u}) \Delta \mathbf{u}=\Delta p \mathbf{q},
$$

where $\mathbf{K}_{T}$ is the tangent stiffness matrix (the second derivative of the strain energy with respect to $\mathbf{u}$ ). Such increments cause out-of-balance forces in Eq. (2). 
Then, in the iterative part, correctors for both $\Delta p$ and $\Delta \mathbf{u}$ are applied iteratively in order to reduce the residual force:

$$
\left\{\begin{array}{l}
\Delta \mathbf{u}_{i+1}=\Delta \mathbf{u}_{i}+\delta \mathbf{u}_{i} \\
\Delta p_{i+1}=\Delta p_{i}+\delta p_{i} .
\end{array}\right.
$$

The subscript $i$ denotes the iteration number within each increment. Various incremental-iterative methods exist. Their differences consist in the respective choices for predictors and correctors. In the following subsection, a powerful predictor-corrector method is briefly reviewed.

\subsection{The arc-length method}

Incremental-iterative methods obtain a series of discrete points along the equilibrium path. In the arc-length approach, a constraint equation is added to Eq. (2):

$$
\left\{\begin{array}{l}
\mathbf{r}(\mathbf{u}, p) \\
L(\mathbf{u}, p)
\end{array}\right\}_{(n+1) \times 1}=\left\{\begin{array}{l}
\mathbf{0} \\
0
\end{array}\right\}_{(n+1) \times 1} .
$$

Here, $n$ represents the number of degrees of freedom (DoFs). In the arc-length method, the additional constraint $L=0$ is chosen as an $(n+1)$ dimensional sphere in the space of $(\mathbf{u}, p) \in \mathbb{R}^{n+1}[29]$ :

$$
\alpha_{1}^{2} \Delta \mathbf{u}^{T} \Delta \mathbf{u}+\alpha_{2}^{2} \Delta p^{2}-\Delta s^{2}=0
$$

where $\Delta s$ is the arc-length, and parameters $\alpha_{1}$ and $\alpha_{2}$ define the contribution of displacement and load terms. $\Delta s / \alpha_{1}$ and $\Delta s / \alpha_{2}$ are the radii of the spherical constraint of Eq. (6) in the directions of $\mathbf{u}$ and $p$, respectively. Figure 1 illustrates the procedure of the arc-length method. This procedure begins from the previous equilibrium point $j$. First, a predictor gives point 1 located on the constraint $L=0$. This jump causes an increase in residual force vector. In order to reduce the out-of-balance force, a corrector procedure is applied to find the next equilibrium point $j+1$. The constraint $L=0$ should be satisfied in all analysis steps.

When the value of $\alpha_{2}$ is set to zero, the second term of Eq. (6) vanishes and the spherical constraint changes into a cylindrical constraint (i.e., the radius of 


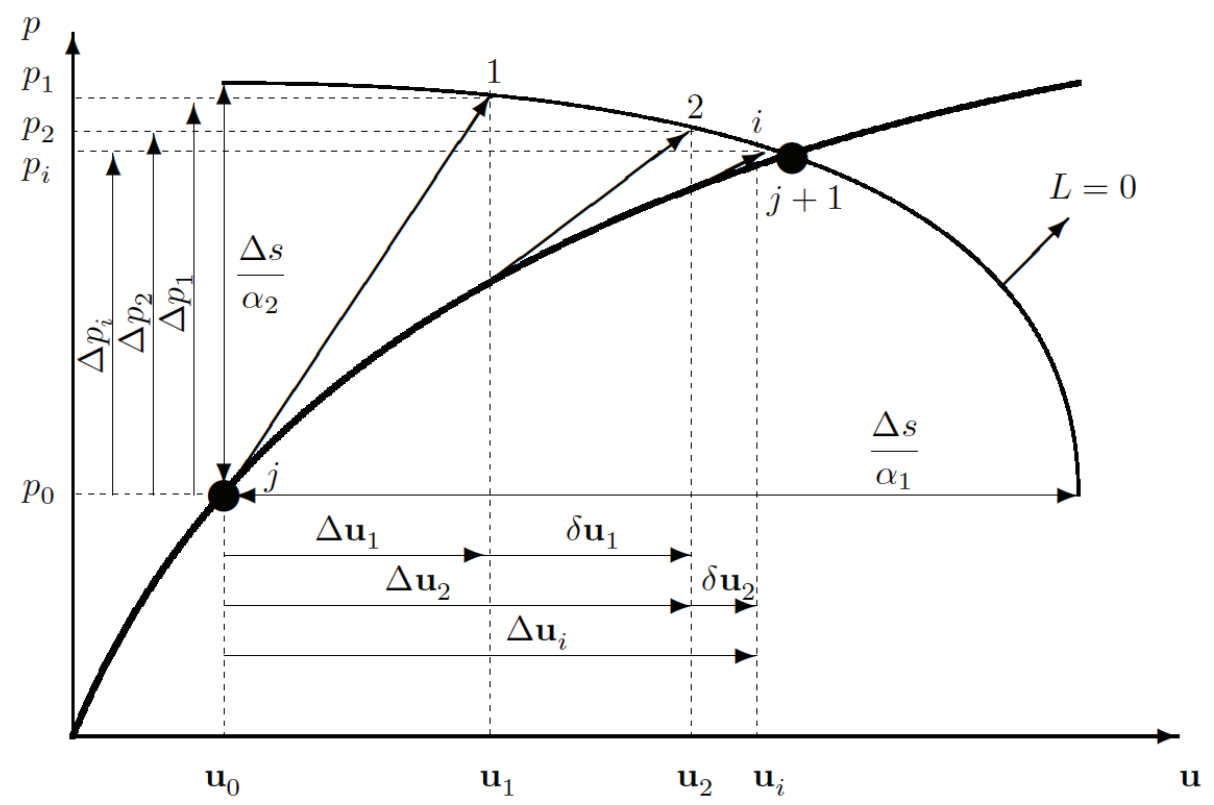

Figure 1: Spherical arc-length procedure

the sphere in the direction of the load parameter $\Delta s / \alpha_{2}$ becomes infinitely large) $[27,30,31]$. Similarly, one can omit the contribution of the displacement term by choosing $\alpha_{1}=0$ and obtain the standard Newton-Raphson (load control) method. According to Figure 1, the first incremental parameters $\Delta \mathbf{u}_{1}$ and $\Delta p_{1}$ are relative to the value of $\Delta s$, and can be computed by the following equations:

$$
\begin{gathered}
\Delta p_{1}=\frac{ \pm \Delta s}{\sqrt{\alpha_{1}^{2} \mathbf{b}_{0}^{T} \mathbf{b}_{0}+\alpha_{2}^{2}}} \\
\Delta \mathbf{u}_{1}=\Delta p_{1} \mathbf{b}_{0} .
\end{gathered}
$$

The subscript 0 denotes the converged values from the previous increment. The correct sign in Eq. (7) is the one that gives the smallest angle between the previous and the current increments [32]. The vectors $\mathbf{a}_{i}$ and $\mathbf{b}_{i}$ are defined as:

$$
\left\{\begin{array}{l}
\mathbf{a}_{i}=-\mathbf{K}_{T i}{ }^{-1} \mathbf{r}_{i} \\
\mathbf{b}_{i}=\mathbf{K}_{T i}{ }^{-1} \mathbf{q} .
\end{array}\right.
$$

After computing the predictors $\Delta \mathbf{u}_{1}$ and $\Delta p_{1}$, iterative correctors follow the 
linearized form of Eq. (5):

$$
\delta \mathbf{u}_{i}=\mathbf{a}_{i}+\delta p_{i} \mathbf{b}_{i}
$$

The arc-length constraint (6) is enforced in each iteration. Therefore, $\delta p_{i}$ will be dependent on $\delta \mathbf{u}_{i}$. By substituting Eqs. (4) and (10) into (6), a quadratic equation for $\delta p_{i}$ is given:

$$
A \delta p_{i}^{2}+B \delta p_{i}+C=0
$$

where

$$
\left\{\begin{array}{l}
A=\alpha_{1}^{2} \mathbf{b}_{i}{ }^{T} \mathbf{b}_{i}+\alpha_{2}^{2} \\
B=2 \alpha_{1}^{2}\left(\Delta \mathbf{u}_{i}+\mathbf{a}_{i}\right)^{T} \mathbf{b}_{i}+2 \alpha_{2}^{2} \Delta p_{i} \\
C=\alpha_{1}^{2}\left(\Delta \mathbf{u}_{i}+\mathbf{a}_{i}\right)^{T}\left(\Delta \mathbf{u}_{i}+\mathbf{a}_{i}\right)+\alpha_{2}^{2} \Delta p_{i}{ }^{2}-\Delta s^{2} .
\end{array}\right.
$$

If two real roots are obtained for $\delta p_{i}$ in (11), the one giving the smallest angle between the previous and the current increments is chosen. If Eq. (11) has complex roots, the value of arc-length $\Delta s$ is reduced [27, 29].

\subsection{Critical points on the equilibrium path}

The tangent stiffness matrix $\mathbf{K}_{T}$ plays an important role in incrementaliterative methods; changes in the characteristics of this matrix can affect the numerical strategy, or lead to degradation of numerical robustness. For instance, this could happen when $\mathbf{K}_{T}$ becomes singular along the equilibrium path. In such cases, the computed values of $\mathbf{a}_{i}$ and $\mathbf{b}_{i}$ in Eq. (9) become infinitely large. These singular points are called critical points. Figure 2 shows three types of critical points. When $\delta p$ is equal to zero, the equilibrium path has a limit point (a). The equilibrium point at the intersection of the initial path with a secondary one is named simple bifurcation point (b). Finally, in the case of multi-bifurcation, more branches intersect (c).

The rank deficiency of $\mathbf{K}_{T}$ is equal to one for limit and simple bifurcation points and two or greater for multi-bifurcation points. The following constraints can be considered for critical points:

$$
\left|\mathbf{K}_{T}\right|=0
$$




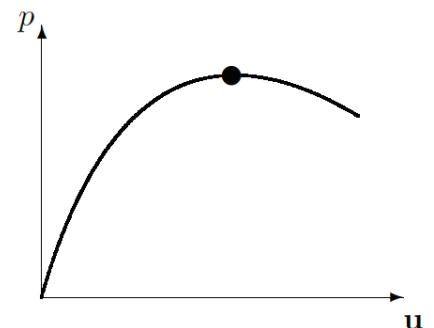

(a)

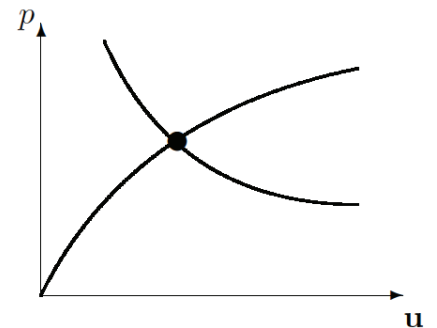

(b)

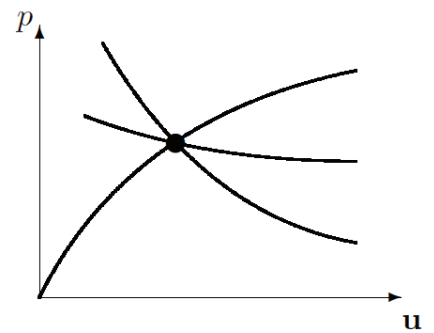

(c)

Figure 2: Critical points: (a) limit (b) simple bifurcation (c) multi-bifurcation

or

$$
\lambda_{k}=0 \quad, \quad k=1, \cdots, h
$$

or

$$
\mathbf{K}_{T} \boldsymbol{\Phi}_{k}=\mathbf{0} \quad, \quad k=1, \cdots, h .
$$

Here, $\lambda_{k}$ and $\boldsymbol{\Phi}_{k}$ represent the $k^{\text {th }}$ critical eigenvalues and eigenvectors of the tangent stiffness matrix, respectively; $h$ is the rank deficiency of $\mathbf{K}_{T}$, and $\left|\mathbf{K}_{T}\right|$ denotes the determinant of stiffness matrix. Since the tangent stiffness matrix is symmetric in conservative systems, the eigenvalues are real, and the left and the right eigenvectors are equal.

\subsection{Direct calculation of critical points}

A critical point satisfies simultaneously the equilibrium equation and belongs to the null space of $\mathbf{K}_{T}$. The direct calculation of critical points has been extensively discussed in the literature $[11,12,13,14,33,34]$. In the following, a direct method for locating multi-bifurcation points is presented that uses the eigenvalue constraint (Eq. (14)). For the iterative solution, both the residual force (2) and the critical constraint (14) are linearized:

$$
\left\{\begin{array}{l}
\mathbf{K}_{T i} \delta \mathbf{u}_{i}-\delta p_{i} \mathbf{q}=-\mathbf{r}_{i} \\
\delta \lambda_{i}=-\lambda_{i}
\end{array}\right.
$$

The subscript $i$ denotes the iteration number within each increment. If the smallest eigenvalue of the tangent stiffness matrix is not a repeated eigenvalue, 
the value of $\delta \lambda_{i}$ can be calculated as follows $[14,15]$ :

$$
\delta \lambda_{i}=\boldsymbol{\Phi}_{i}^{T} \delta \mathbf{K}_{T i} \boldsymbol{\Phi}_{i}=\boldsymbol{\Phi}_{i}^{T}\left(\delta \mathbf{u}_{i} \frac{\partial \mathbf{K}_{T i}}{\partial \mathbf{u}}\right) \boldsymbol{\Phi}_{i} .
$$

Here, $\boldsymbol{\Phi}_{i}$ is the eigenvector corresponding to the smallest eigenvalue in the $i^{\text {th }}$ iteration. In the case of repeated eigenvalues, the minimum variation in $\delta \lambda_{i}$ due to $\delta \mathbf{u}_{i}$ can be written in the following form [35]:

$$
\delta \lambda_{i}=\mu \boldsymbol{\Psi}_{i}^{T}\left(\delta \mathbf{u}_{i} \frac{\partial \mathbf{K}_{T i}}{\partial \mathbf{u}}\right) \boldsymbol{\Psi}_{i},
$$

where $\mu$ represents the minimum eigenvalue of the matrix, and each column of $\boldsymbol{\Psi}_{i}$ includes the normalized eigenvector relative to the smallest eigenvalues in the $i^{\text {th }}$ iteration. Therefore, the matrix $\boldsymbol{\Psi}_{i}$ has $h$ columns. Finding the smallest eigenvalues and the relative eigenvectors can be done by standard methods (e.g., Rayleigh quotient iteration, Lanczos algorithm or inverse power method). By substituting Eqs. (10) into (18), we obtain a relationship between $\delta \lambda_{i}$ and $\delta p_{i}$ :

$$
\delta \lambda_{i}=\mu \boldsymbol{\Psi}_{i}^{T}\left(\delta \mathbf{K}_{\mathbf{a} i}+\delta p_{i} \delta \mathbf{K}_{\mathbf{b} i}\right) \boldsymbol{\Psi}_{i} .
$$

The matrices $\delta \mathbf{K}_{\mathbf{a} i}$ and $\delta \mathbf{K}_{\mathbf{b} i}$ denote the directional derivatives of $\mathbf{K}_{T i}$ with respect to the vectors $\mathbf{a}_{i}$ and $\mathbf{b}_{i}$, respectively. In order to calculate derivatives of the tangent stiffness matrix numerically, the following approximations are helpful when $t$ is sufficiently small:

$$
\begin{aligned}
\delta \mathbf{K}_{\mathbf{a}} & =\frac{\mathbf{K}_{T}(\mathbf{u}+t \mathbf{a})-\mathbf{K}_{T}(\mathbf{u})}{t} \\
\delta \mathbf{K}_{\mathbf{b}} & =\frac{\mathbf{K}_{T}(\mathbf{u}+t \mathbf{b})-\mathbf{K}_{T}(\mathbf{u})}{t} .
\end{aligned}
$$

By substituting (19) into the second equation of (16), we obtain the value of $\delta p_{i}$ in the current iteration. $\delta \mathbf{u}_{i}$ is available from the first equation of (16). The values of $\mathbf{u}_{i}$ and $p_{i}$ are then updated for the next iteration.

In conservative systems, the tangent stiffness matrix $\mathbf{K}_{T}$ is symmetric. Consequently, it can be expressed as

$$
\mathbf{K}_{T}=\sum_{k=1}^{n} \lambda_{k} \boldsymbol{\Phi}_{k} \boldsymbol{\Phi}_{k}^{T}
$$


and subsequently, its inverse is

$$
\mathbf{K}_{T}{ }^{-1}=\sum_{k=1}^{n} \frac{1}{\lambda_{k}} \boldsymbol{\Phi}_{k} \boldsymbol{\Phi}_{k}^{T} .
$$

At critical points, the tangent stiffness matrix is singular. As a result, an approximation of $\mathbf{K}_{T}^{-1}$ is needed in the neighbourhood of such points where the magnitude of $1 / \lambda_{k}$ significantly increases when the value of critical $\lambda_{k}$ is near zero. This increase can cause a large numerical error and sometimes, divergence in the iterative method. To overcome this, the tangent stiffness matrix can be modified [14, 36]:

$$
\overline{\mathbf{K}}_{T}=\mathbf{K}_{T}+\sum_{k=1}^{h} \lambda_{k}^{*} \boldsymbol{\Phi}_{k} \boldsymbol{\Phi}_{k}^{T},
$$

where $\lambda_{k}^{*}$ are artificial stiffnesses, $h$ is the rank deficiency of the matrix, and $\boldsymbol{\Phi}_{k}$ are eigenvectors corresponding to null eigenvalues.

The authors' experience shows that the choice of $\lambda_{k}^{*}$ and $t$ can have a strong influence on the asymptotic convergence rate. The smallest possible values (depending on the precision used in the computer programming) for these parameters lead to better results.

\section{Equilibrium and critical state for parameterized systems}

The load-bearing capacity of real structures is usually affected by parameters, such as geometric defects, load imperfections and thermal stresses that can occur before or during the structural loading. In this section, governing equations for conservative systems including control parameters are given.

In parameterized elastic structures, the total potential energy $\Pi$ is a function of the displacement $\mathbf{u} \in \mathbb{R}^{n}$, the load parameter $p \in \mathbb{R}$ and an additional control parameter with magnitude $\varepsilon \in \mathbb{R}$. For all conservative systems, $\Pi$ is constant for all equilibrium states $[3,19,26]$ :

$$
\Pi(\mathbf{u}, p, \varepsilon)=\text { constant. }
$$

In order to obtain the governing equilibrium equations, the first derivative of $\Pi$ 
with respect to $\mathbf{u}$ is set to zero:

$$
\Pi_{\mathbf{u}}(\mathbf{u}, p, \varepsilon)=\mathbf{r}(\mathbf{u}, p, \varepsilon)=\mathbf{0}
$$

Here, the subscript $\mathbf{u}$ represents the derivative with respect to the nodal displacement, and $\mathbf{r}$ is the residual force. For a structure with $n$ degrees of freedom, (26) is a system of $n$ equations with $n+2$ unknowns. Consequently, the equilibrium manifold, i.e., the set of points satisfying (26), is composed by one or more surfaces in the space of $(\mathbf{u}, p, \varepsilon) \in \mathbb{R}^{n+2}$ (Figure 3 ).

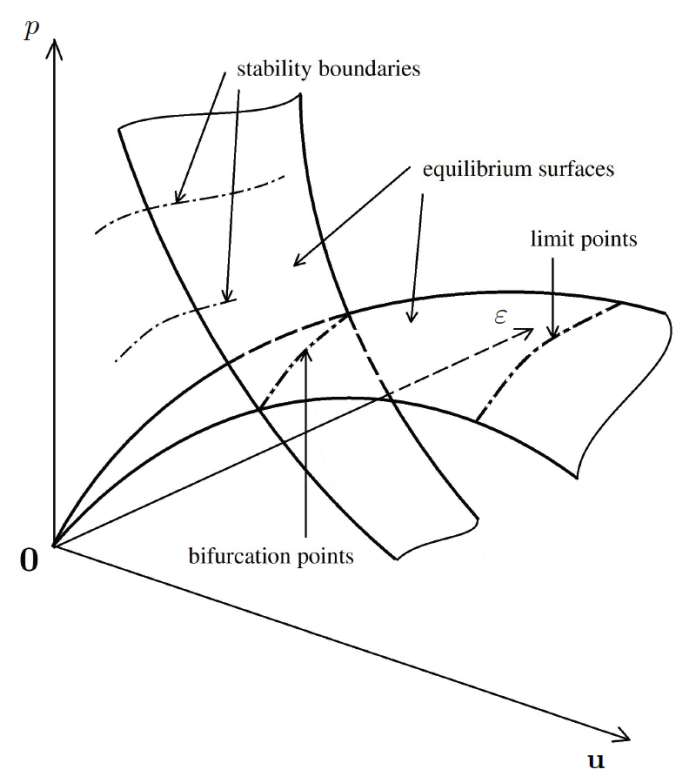

Figure 3: Equilibrium surfaces, critical points and stability boundaries in a parameterized system

Similar to perfect structures, there could be critical points on the equilibrium surface. Figure 3 displays both limit and bifurcation points in the space of $(\mathbf{u}, p, \varepsilon)$ for an imperfect system. At limit points, $\partial p / \partial \mathbf{u}=\mathbf{0}$ on the equilibrium surface; bifurcation points are generated when two or more equilibrium surfaces intersect. In both cases, the second derivative of the total potential energy with respect to $\mathbf{u}$ is singular:

$$
\left|\Pi_{\mathbf{u u}}(\mathbf{u}, p, \varepsilon)\right|=0 .
$$


By considering the $n+1$ equations of (26) and (27), one can conclude that the set of critical points lies on a curve or a number of curves in the space of $(\mathbf{u}, p, \varepsilon)$. The projection of critical points onto the plane of $p$ and $\varepsilon$ axes is called stability boundary (Figure 3).

If $\Pi(\mathbf{u}, p, \varepsilon)$ is a summation of the internal strain energy and the external work done by the displacement independent loads, the second derivative of the total potential energy is equal to $\mathbf{K}_{T}$. At the stability boundary of imperfect structures, the following equations should be satisfied:

$$
\left\{\begin{array}{c}
\mathbf{r}(\mathbf{u}, p, \varepsilon) \\
\mathbf{G}(\mathbf{u}, \varepsilon) \\
L(\mathbf{u}, p, \varepsilon)
\end{array}\right\}=\left\{\begin{array}{l}
\mathbf{0} \\
\mathbf{0} \\
0
\end{array}\right\},
$$

where $\mathbf{G}$ is the critical condition, and $L$ represents the additional constraint needed in incremental-iterative strategies. One way to define $\mathbf{G}$ for imperfect structures is based on the existence of (a few) eigenvectors corresponding to the null space of the tangent stiffness matrix $[13,37]$. Subsequently, for simple critical points, the critical condition can be written as [38, 39]:

$$
\mathbf{G}(\mathbf{u}, \varepsilon)=\left\{\begin{array}{c}
\mathbf{K}_{T}(\mathbf{u}, \varepsilon) \mathbf{\Phi} \\
l(\boldsymbol{\Phi})
\end{array}\right\} .
$$

Here, $l(\mathbf{\Phi})$ denotes a normalizing function removing the singularity of the system (28). In the case of multiple criticality, the same constraint (29) can be considered for each critical eigenvector [39]. In the literature, the sensitivity analysis of structures (done by incremental-iterative procedures) is investigated mostly by using the critical condition (29) (see, for example, [2, 40, 41, 42]). A weakness of this strategy is that the obtained critical state is not necessarily the first critical point on the equilibrium path. The authors' experience shows that when a number of critical points are close to each other, this method is sensitive to the initial guess of the critical eigenvector, and may cause the method to converge to the second, third or further critical point on the equilibrium path. As a result, important critical states may not be identified during the analysis of complex structures. In the next section, a numerical procedure to trace the 
stability boundary is introduced. This procedure deals with critical eigenvalues of the tangent stiffness matrix instead of the constraint (29), and does not have the drawback mentioned above.

\section{Stability boundary}

In the previous section, it is explained that the stability boundary is the projection of critical points onto the $p-\varepsilon$ plane, and it shows the relation between load-bearing capacity and the magnitude of the control parameter. In the following, a direct numerical method is introduced to trace the stability boundary. It is able to track limit, simple and multi-bifurcation points. This method is based on an incremental-iterative algorithm and exhibits a quadratic rate of convergence. The proposed technique is capable to track the stability boundary for a wide range of the control parameter.

\subsection{Formulation of an incremental-iterative method for two-parameter systems}

Critical points are equilibrium points for which the tangent stiffness matrix is singular. Since they belong to the equilibrium manifold, the residual force is zero:

$$
\mathbf{r}(\mathbf{u}, p, \varepsilon)=\mathbf{0} .
$$

The linearized form of Eq. (30) at the step $i$ can be written as

$$
\frac{\partial \mathbf{r}_{i}}{\partial \mathbf{u}} \delta \mathbf{u}_{i}+\frac{\partial \mathbf{r}_{i}}{\partial p} \delta p_{i}+\frac{\partial \mathbf{r}_{i}}{\partial \varepsilon} \delta \varepsilon_{i}=-\mathbf{r}_{i}
$$

where $\mathbf{K}_{T}=\partial \mathbf{r} / \partial \mathbf{u}$ and $\mathbf{q}=-\partial \mathbf{r} / \partial p$ are, respectively, the tangent stiffness matrix and the reference load vector in imperfect elastic structures. For structures under displacement independent loads, the vector $\mathbf{q}$ is constant. The term $\partial \mathbf{r} / \partial \varepsilon$ is an additional force vector $\mathbf{F}_{\varepsilon}(\mathbf{u}, \varepsilon)$ due to the control parameter. The value of $\mathbf{F}_{\varepsilon}$ for sufficiently small $t$ can be estimated by

$$
\mathbf{F}_{\varepsilon}=\frac{\mathbf{r}(\mathbf{u}, p, \varepsilon+t)-\mathbf{r}(\mathbf{u}, p, \varepsilon)}{t} .
$$

For some typical control parameters, the exact value of $\mathbf{F}_{\varepsilon}$ is available. For instance, in the case of elastic structures with an extra loading, $\mathbf{F}_{\varepsilon}$ equals the 
negative of the additional load vector. $\mathbf{F}_{\varepsilon}$ is equivalent to a thermal force vector when variation in temperature causes thermal stresses in members. In the particular case of initial geometric defects in structures with small strains, $\mathbf{F}_{\varepsilon}$ is equivalent to an additional force vector that deforms the perfect structure to the imperfect state. Examples of such control parameters will be discussed in the numerical examples section.

Similar to other incremental-iterative algorithms, the proposed method has two parts. Starting from an existing critical point, we first calculate the predictors $\Delta \mathbf{u}_{1}, \Delta p_{1}$ and $\Delta \varepsilon_{1}$ determining the step size to the next critical point. The latter part updates these increments in each iteration by applying the correctors $\delta \mathbf{u}_{i}, \delta p_{i}$ and $\delta \varepsilon_{i}$. After calculating the residual force and its derivatives in Eq. (31), we can obtain the following relationship:

$$
\delta \mathbf{u}_{i}=\mathbf{a}_{i}+\delta p_{i} \mathbf{b}_{i}+\delta \varepsilon_{i} \mathbf{c}_{i}
$$

where

$$
\left\{\begin{array}{l}
\mathbf{a}_{i}=-\mathbf{K}_{T i}{ }^{-1} \mathbf{r}_{i} \\
\mathbf{b}_{i}=\mathbf{K}_{T i}{ }^{-1} \mathbf{q} \\
\mathbf{c}_{i}=-\mathbf{K}_{T i}{ }^{-1} \mathbf{F}_{\varepsilon i} .
\end{array}\right.
$$

Eq. (24) is used to compute the inverse of $\mathbf{K}_{T}$ in the vicinity of critical points.

The other constraint that should be satisfied along the stability boundary is the singularity of the tangent stiffness matrix (one or more eigenvalues of $\mathbf{K}_{T}$ are zero):

$$
\lambda_{k}=0 \quad, \quad k=1, \cdots, h
$$

According to [35], the minimum variation of the smallest eigenvalue(s) with respect to a variation in tangent stiffness matrix $\delta \mathbf{K}_{T i}$ is

$$
\delta \lambda_{i}=\mu \mathbf{\Psi}_{i}^{T} \delta \mathbf{K}_{T i} \boldsymbol{\Psi}_{i}
$$

where $\mu$ is the minimum eigenvalue of the matrix, and the columns of $\boldsymbol{\Psi}_{i}$ are the normalized eigenvectors relative to the repeated smallest eigenvalue (only $h$ eigenvectors are required). For an eigenvalue with multiplicity one, $\boldsymbol{\Psi}_{i}$ has 
one column. Since the tangent stiffness is a function of $\mathbf{u}$ and $\varepsilon$, Eq. (36) is rewritten as

$$
\delta \lambda_{i}=\mu \mathbf{\Psi}_{i}^{T}\left(\delta \mathbf{u}_{i} \frac{\partial \mathbf{K}_{T i}}{\partial \mathbf{u}}+\delta \varepsilon_{i} \frac{\partial \mathbf{K}_{T i}}{\partial \varepsilon}\right) \mathbf{\Psi}_{i} .
$$

By substituting Eqs. (33) into (37), we obtain the value of $\delta \lambda_{i}$ as a function of $\delta p_{i}$ and $\delta \varepsilon_{i}$ :

$$
\delta \lambda_{i}=\mu \mathbf{\Psi}_{i}^{T}\left(\delta \mathbf{K}_{\mathbf{a} i}+\delta p_{i} \delta \mathbf{K}_{\mathbf{b}_{i}}+\delta \varepsilon_{i}\left(\delta \mathbf{K}_{\mathbf{c} i}+\delta \mathbf{K}_{\varepsilon i}\right)\right) \boldsymbol{\Psi}_{i},
$$

where $\delta \mathbf{K}_{\mathbf{a} i}, \delta \mathbf{K}_{\mathbf{b} i}$, and $\delta \mathbf{K}_{\mathbf{c} i}$ are, respectively, directional derivatives with respect to vectors $\mathbf{a}_{i}, \mathbf{b}_{i}$, and $\mathbf{c}_{i}$. The matrix $\delta \mathbf{K}_{\varepsilon i}$ denotes the derivative of $\mathbf{K}_{T i}$ with respect to the control parameter $\varepsilon$. When $t$ is sufficiently small, derivatives of the tangent stiffness matrix in (38) can be approximated:

$$
\left\{\begin{array}{l}
\delta \mathbf{K}_{\mathbf{a}}=\frac{1}{t}\left(\mathbf{K}_{T}(\mathbf{u}+t \mathbf{a}, \varepsilon)-\mathbf{K}_{T}(\mathbf{u}, \varepsilon)\right) \\
\delta \mathbf{K}_{\mathbf{b}}=\frac{1}{t}\left(\mathbf{K}_{T}(\mathbf{u}+t \mathbf{b}, \varepsilon)-\mathbf{K}_{T}(\mathbf{u}, \varepsilon)\right) \\
\delta \mathbf{K}_{\mathbf{c}}=\frac{1}{t}\left(\mathbf{K}_{T}(\mathbf{u}+t \mathbf{c}, \varepsilon)-\mathbf{K}_{T}(\mathbf{u}, \varepsilon)\right) \\
\delta \mathbf{K}_{\varepsilon}=\frac{1}{t}\left(\mathbf{K}_{T}(\mathbf{u}, \varepsilon+t)-\mathbf{K}_{T}(\mathbf{u}, \varepsilon)\right) .
\end{array}\right.
$$

By comparing Eqs. (36) and (38), it can be concluded that $\delta \mathbf{K}_{T i}$ is a summation of three matrices. Since variations in eigenvectors of the tangent stiffness matrix due to $\delta \mathbf{K}_{T i}$ are negligible along each iteration, one can rewrite the right hand side of Eq. (38) as a linear combination of the terms $\mu \mathbf{\Psi}_{i}{ }^{T} \delta \mathbf{K}_{\mathbf{a} i} \mathbf{\Psi}_{i}$, $\mu \boldsymbol{\Psi}_{i}{ }^{T} \delta \mathbf{K}_{\mathbf{b} i} \boldsymbol{\Psi}_{i}$ and $\mu \boldsymbol{\Psi}_{i}{ }^{T}\left(\delta \mathbf{K}_{\mathbf{c} i}+\delta \mathbf{K}_{\varepsilon i}\right) \boldsymbol{\Psi}_{i}$. From this simplification and from the linearization of Eq. (35) $\left(\delta \lambda_{i}=-\lambda_{i}\right)$, we derive a relationship between the correctors $\delta p_{i}$ and $\delta \varepsilon_{i}$ :

$$
\delta p_{i}=a_{i}^{\prime}+\delta \varepsilon_{i} b_{i}^{\prime}
$$

where

$$
\left\{\begin{array}{c}
a_{i}^{\prime}=-\frac{\lambda_{i}+\mu \mathbf{\Psi}_{i}^{T} \delta \mathbf{K}_{\mathbf{a} i} \mathbf{\Psi}_{i}}{\mu \mathbf{\Psi}_{i}^{T} \delta \mathbf{K}_{\mathbf{b} i} \mathbf{\Psi}_{i}} \\
b_{i}^{\prime}=-\frac{\mu \mathbf{\Psi}_{i}^{T}\left(\delta \mathbf{K}_{\mathbf{c} i}+\delta \mathbf{K}_{\varepsilon i}\right) \boldsymbol{\Psi}_{i}}{\mu \mathbf{\Psi}_{i}^{T} \delta \mathbf{K}_{\mathbf{b} i} \mathbf{\Psi}_{i}} .
\end{array}\right.
$$

By comparing Eqs. (33) and (40), one can obtain the following equation:

$$
\delta \mathbf{u}_{i}=\mathbf{a}_{i}{ }^{\prime \prime}+\delta \varepsilon_{i} \mathbf{b}_{i}{ }^{\prime \prime},
$$


where

$$
\left\{\begin{array}{l}
\mathbf{a}_{i}{ }^{\prime \prime}=\mathbf{a}_{i}+a_{i}^{\prime} \mathbf{b}_{i} \\
\mathbf{b}_{i}{ }^{\prime \prime}=b_{i}^{\prime} \mathbf{b}_{i}+\mathbf{c}_{i} .
\end{array}\right.
$$

In order to trace the curve of critical points in the space of $(\mathbf{u}, p, \varepsilon) \in \mathbb{R}^{n+2}$, an additional constraint is needed. In this paper, the authors suggest an $(n+2)$ dimensional sphere as a constraint:

$$
\alpha_{1}^{2} \Delta \mathbf{u}^{T} \Delta \mathbf{u}+\alpha_{2}^{2} \Delta p^{2}+\alpha_{3}^{2} \Delta \varepsilon^{2}-\Delta s^{2}=0
$$

Here, $\Delta s$ is called the arc-length, and the parameters $\alpha_{1}, \alpha_{2}$ and $\alpha_{3}$ control the scaling between displacement, load and control parameter terms. Similar to the arc-length method described before, $\Delta s / \alpha_{1}, \Delta s / \alpha_{2}$ and $\Delta s / \alpha_{3}$ are the radii of the spherical constraint Eq. (44) in the directions of $\mathbf{u}, p$ and $\varepsilon$, respectively. By substituting the updated values of $\Delta \mathbf{u}, \Delta p$ and $\Delta \varepsilon$ in Eq. (44), we obtain a quadratic equation for $\delta \varepsilon_{i}$ :

$$
A \delta \varepsilon_{i}^{2}+B \delta \varepsilon_{i}+C=0
$$

where

$$
\left\{\begin{array}{l}
A=\alpha_{1}^{2} \mathbf{b}_{i}{ }^{\prime \prime T} \mathbf{b}_{i}{ }^{\prime \prime}+\alpha_{2}^{2} b_{i}^{\prime 2}+\alpha_{3}^{2} \\
B=2 \alpha_{1}^{2}\left(\Delta \mathbf{u}_{i}+\mathbf{a}_{i}{ }^{\prime \prime}\right)^{T} \mathbf{b}_{i}{ }^{\prime \prime}+2 \alpha_{2}^{2}\left(\Delta p_{i}+a_{i}^{\prime}\right) b_{i}^{\prime}+2 \alpha_{3}^{2} \Delta \varepsilon_{i} \\
C=\alpha_{1}^{2}\left(\Delta \mathbf{u}_{i}+\mathbf{a}_{i}{ }^{\prime \prime}\right)^{T}\left(\Delta \mathbf{u}_{i}+\mathbf{a}_{i}{ }^{\prime \prime}\right)+\alpha_{2}^{2}\left(\Delta p_{i}+a_{i}^{\prime}\right)^{2}+\alpha_{3}^{2} \Delta \varepsilon_{i}{ }^{2}-\Delta s^{2} .
\end{array}\right.
$$

When (45) has two real values for $\delta \varepsilon_{i}$, the one that gives the minimum angle between the previous and the current increments is chosen. If Eq. (45) has complex roots, a reduction in the magnitude of the arc-length $\Delta s$ is needed.

Since the constraint (44) should be satisfied throughout the whole sequence, the predictors $\Delta \mathbf{u}_{1}, \Delta p_{1}$ and $\Delta \varepsilon_{1}$ at the first step are related to the value of $\Delta s:$

$$
\begin{gathered}
\Delta \varepsilon_{1}=\frac{ \pm \Delta s}{\sqrt{\alpha_{1}^{2} \mathbf{b}_{0}{ }^{\prime \prime} \mathbf{b}_{0}{ }^{\prime \prime}+\alpha_{2}^{2} b_{0}^{\prime 2}+\alpha_{3}^{2}}} \\
\Delta p_{1}=\Delta \varepsilon_{1} b_{0}^{\prime}
\end{gathered}
$$




$$
\Delta \mathbf{u}_{1}=\Delta \varepsilon_{1} \mathbf{b}_{0}{ }^{\prime \prime}
$$

We choose the sign of $\Delta s$ in Eq. (47) depending on the direction of the previous increment. The correct solution is the one that gives the smallest angle between the previous and the current increments [32].

Note that the method we present is based on Newton's scheme, and it has the expected quadratic convergence behaviour [10, 13], which will be demonstrated in Section 5. In addition, since the proposed method is an incremental-iterative procedure, and each critical point is directly computed from the previous one, it can trace the stability boundary for a wide range of the control parameter $\varepsilon$ without using globalization techniques (such as those described in [14, 15, 43]). The number of multiple bifurcation points is usually very limited throughout the stability boundary (see the numerical examples in Section 5). As a result, in most incremental and iterative steps, we only need to compute a single critical eigenvector relative to the smallest eigenvalue to obtain the coefficients $a_{i}^{\prime}$ and $b_{i}^{\prime}$ in Eq. (41). When a multiple bifurcation point is passed in an increment or iteration, the calculation of additional critical eigenvectors is needed in that step. In this state, the number of critical eigenvectors is related to the multiplicity of the null eigenvalue.

Similar to equilibrium paths, stability boundaries can also have bifurcations (see, for instance, the numerical examples in Section 5). The capability of choosing the desired branch is an advantage of the proposed procedure. According to Eq. (36), since the variation of the smallest eigenvalue(s) is considered, the proposed method automatically traces the stability boundary corresponding to the first critical point on the equilibrium path. The locus of the second, third or further critical points will be obtained, when the eigenvectors relative to the former critical modes are omitted from $\boldsymbol{\Psi}_{i}$. In other words, by using the proposed method, all branches of stability boundaries can be traced, while most strategies based on the eigenvector constraint (29) only follow the fundamental stability boundary, and, without additional detecting functions, miss the presence of other branches. 


\subsection{Numerical implementation}

The proposed method is able to trace the stability boundary from an initial critical point without considering the pre-critical equilibrium path. In the following, the computational steps of the predictor (incremental) and corrector (iterative) steps are given:

\section{Incremental part}

1. Prediction of the arc-length $\Delta s$ : The magnitude of $\Delta s$ can be adjusted/chosen based on the number of iterations and the length of increments in previous steps.

2. Calculation of $b_{0}^{\prime}$ and $\mathbf{b}_{0}{ }^{\prime \prime}$ from Eqs. (41) and (43).

3. Estimation of $\alpha_{1}, \alpha_{2}$ and $\alpha_{3}$ : Based on the current characteristics of the system, the contribution of $\Delta \mathbf{u}_{1}, \Delta p_{1}$ and $\Delta \varepsilon_{1}$ in the arc-length $\Delta s$ could be changed in each increment. This contribution is modified by the proportions of $\alpha_{1}, \alpha_{2}$ and $\alpha_{3}$. Let

$$
\left\{\begin{array}{l}
\alpha_{1}=\left\|\mathbf{b}_{0}{ }^{\prime \prime}\right\| / \Delta \\
\alpha_{2}=\left|b_{0}^{\prime}\right| / \Delta \\
\alpha_{3}=1 / \Delta,
\end{array}\right.
$$

where

$$
\Delta=\sqrt{\mathbf{b}_{0}{ }^{\prime T} \mathbf{b}_{0}{ }^{\prime \prime}+b_{0}^{\prime 2}+1} .
$$

Eq. (50) is only an example of possible contributions from $\alpha_{i}$. In our experience, this choice ensures a robust and efficient simulation. This choice is equivalent to saying that the contribution of each term in Eq. (44) depends on the cosine of the angle between the tangent of stability boundary and the axes $\mathbf{u}, p$ and $\varepsilon$. A smaller angle gives a higher contribution.

4. Calculation of $\Delta \mathbf{u}_{1}, \Delta p_{1}$ and $\Delta \varepsilon_{1}$ : These predictors are derived from Eqs. (47)-(49). 


\section{Iterative part}

5. Computation of the residual force $\mathbf{r}_{i}$ and of the additional force vector $\mathbf{F}_{\varepsilon i}$.

6. Determination of $a_{i}^{\prime}, b_{i}^{\prime}, \mathbf{a}_{i}{ }^{\prime \prime}$ and $\mathbf{b}_{i}{ }^{\prime \prime}$ from Eqs. (41) and (43).

7. Solution of the quadratic equation (45): To obtain a solution for Eq. (45), first, the coefficients of $A, B$ and $C$ are computed from Eq. (46). If the sign of $B^{2}-4 A C$ is negative (complex roots), the procedure will stop and restart from Step 1 with a reduced $\Delta s$. If two real roots are obtained, the one that gives the smallest angle between the previous and the current increments is selected.

8. Calculation of the correctors $\delta p_{i}$ and $\delta \mathbf{u}_{i}$ from Eqs. (40) and (42), respectively.

9. Update of the increments.

10. Verification of the convergence criterion. In this paper, two convergence criteria are applied: (i) energy $\left(\left|\delta \mathbf{u}_{i}{ }^{T} \mathbf{r}_{i}\right| \leq \beta_{e}\left|\Delta \mathbf{u}_{1}{ }^{T} \mathbf{r}_{1}\right|\right)$, and (ii) magnitude of the minimum eigenvalue $\left(\left|\lambda_{i}\right| \leq \beta_{\lambda}\left|\lambda_{1}\right|\right)$. $\beta_{e}$ and $\beta_{\lambda}$ are the tolerances.

In order to find the first critical point, one can use the indirect computation of critical points method [44]. For this purpose, a test function is defined and evaluated when tracing the equilibrium path. A change in the sign of this function (from an equilibrium point to the next one) shows that a critical point has been passed (bracketing procedure). The most effective and accurate but also more costly test function for all types of critical points is the smallest eigenvalue of the tangent stiffness matrix [44]. To compute the smallest eigenvalues, standard methods, like Rayleigh quotient iteration, Lanczos algorithm or inverse power method, can be used. The next step consists in finding a critical point as a starting point for tracing the stability boundary. This point can be obtained by (1) finding the zero value for the test function [44], or (2) by means of the "Iterative part" mentioned above. 


\section{Numerical examples}

An algorithm to obtain the stability boundary has been described in the previous section. In this section, several examples show the ability of this method to directly evaluate the critical points for non-linear elastic structures and prove its computational efficiency. The relationship between strains and displacements is defined in terms of the Green's strain. The convergence tolerances are $\beta_{e}=10^{-16}$ (energy) and $\beta_{\lambda}=10^{-8}$ (minimum eigenvalue magnitude). As it is mentioned in Section 2, the choice of $\lambda_{k}^{*}$ and $t$ can have a strong effect on the asymptotic convergence rate. Smaller values (depending on the precision used in computer programming) lead to better results. For best performance (e.g. convergence rate), $\lambda_{k}^{*}$ and $t$ are, respectively, assumed $10^{-32}$ and $10^{-64}$ for all examples. All calculations were performed with the software Wolfram Mathematica 8.0, which allows detailed control over precision and accuracy [45]. Larger values for $\lambda_{k}^{*}$ and $t$ can be used, the only consequence being a slight degradation of the convergence rate. The magnitude of the arc-length $\Delta s$ is constant for incremental steps unless Eq. (45) has complex roots. In this case, the value of $\Delta s$ is adaptively reduced until Eq. (45) has real roots. The choice of $\Delta s$ has an effect on the distance between the obtained critical points and the resolution of the representation of the stability boundary. Here, the choice for $\Delta s$ is based on the range of the control parameter for each example. The Rayleigh quotient iteration is employed for computing the smallest (and sometimes repeated) eigenvalue and its relative eigenvector(s). This iterative technique has cubic convergence $[14,15]$. For all examples, we provide graphical representations of the stability boundary and tabulated data with additional information (e.g., parameter values, numerical performance).

\subsection{Truss-spring system}

The simple truss-spring system (Figure 4) was investigated by several authors $[28,46,47]$. The perfect structure consists of two truss bars in the $x-y$ plane, laterally supported by a horizontal spring in the $z$ direction. By increasing the external load $P$, the equilibrium path reaches a limit point. In this 
example, an initial geometrical parameter $\varepsilon$ is considered that describes the position of the top node in the direction of $z$. The height of the truss-spring system is $h$, and $2 L$ represents the span. $E A$ and $k$ are the stiffnesses of the bars and the spring, respectively. In this example, $k L / E A=0.02$ and $h / L=0.2$.

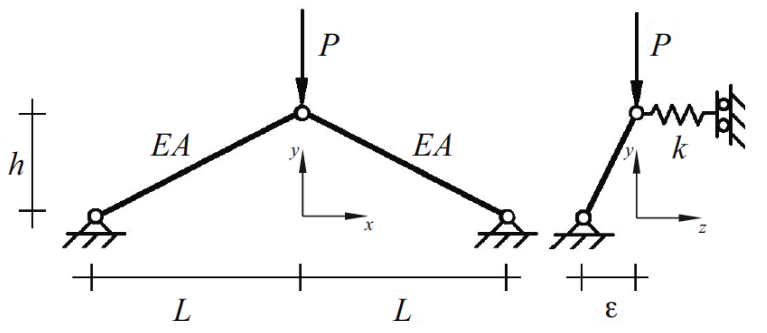

Figure 4: Truss-spring system

For the perfect structure $(\varepsilon / h=0)$, the ratio of $P_{c r} / E A$ is equal to $2.741 \times 10^{-3}$. The proposed algorithm was applied to trace the stability boundary for a structure with different out of plane deviations $\varepsilon / h$. Figure 5 illustrates the effect of the control parameter on the critical load. For this analysis, $\Delta s=0.04$ and the values of $\alpha_{i}$ are updated in each increment based on Eq. (50). As it can be seen, the critical points given by [28] are consistent with the stability boundary calculated by the proposed method. The summary of computations is given in Table 1.

Table 1: Analysis of the truss-spring system

\begin{tabular}{|c|c|c|c|c|c|c|}
\hline \multirow{2}{*}{ Increment } & \multirow{2}{*}{$\begin{array}{c}\text { No. of } \\
\text { iterations }\end{array}$} & \multicolumn{3}{|c|}{ Contribution of $\alpha_{i}$} & \multirow{2}{*}{$\begin{array}{c}\text { Control Par. } \\
\varepsilon / h\end{array}$} & \multirow{2}{*}{$\begin{array}{c}\text { Critical load } \\
P_{c r} / E A\end{array}$} \\
\hline & & $\alpha_{1}$ & $\alpha_{2}$ & $\alpha_{3}$ & & \\
\hline 1 & 4 & $1.000 \times 10^{0}$ & $3.809 \times 10^{-64}$ & $4.433 \times 10^{-8}$ & $2.558 \times 10^{-2}$ & $2.462 \times 10^{-3}$ \\
\hline 2 & 4 & $9.525 \times 10^{-1}$ & $1.142 \times 10^{-2}$ & $3.042 \times 10^{-1}$ & $1.364 \times 10^{-1}$ & $1.880 \times 10^{-3}$ \\
\hline 3 & 4 & $8.060 \times 10^{-1}$ & $1.191 \times 10^{-2}$ & $5.918 \times 10^{-1}$ & $3.200 \times 10^{-1}$ & $1.313 \times 10^{-3}$ \\
\hline 4 & 4 & $7.078 \times 10^{-1}$ & $8.447 \times 10^{-3}$ & $7.064 \times 10^{-1}$ & $5.257 \times 10^{-1}$ & $9.221 \times 10^{-4}$ \\
\hline 5 & 4 & $6.720 \times 10^{-1}$ & $5.553 \times 10^{-3}$ & $7.405 \times 10^{-1}$ & $7.355 \times 10^{-1}$ & $6.667 \times 10^{-4}$ \\
\hline 6 & 3 & $6.633 \times 10^{-1}$ & $3.666 \times 10^{-3}$ & $7.484 \times 10^{-1}$ & $9.456 \times 10^{-1}$ & $4.969 \times 10^{-4}$ \\
\hline 7 & 3 & $6.645 \times 10^{-1}$ & $2.473 \times 10^{-3}$ & $7.473 \times 10^{-1}$ & $1.155 \times 10^{0}$ & $3.807 \times 10^{-4}$ \\
\hline 8 & 3 & $6.689 \times 10^{-1}$ & $1.714 \times 10^{-3}$ & $7.433 \times 10^{-1}$ & $1.364 \times 10^{0}$ & $2.991 \times 10^{-4}$ \\
\hline
\end{tabular}




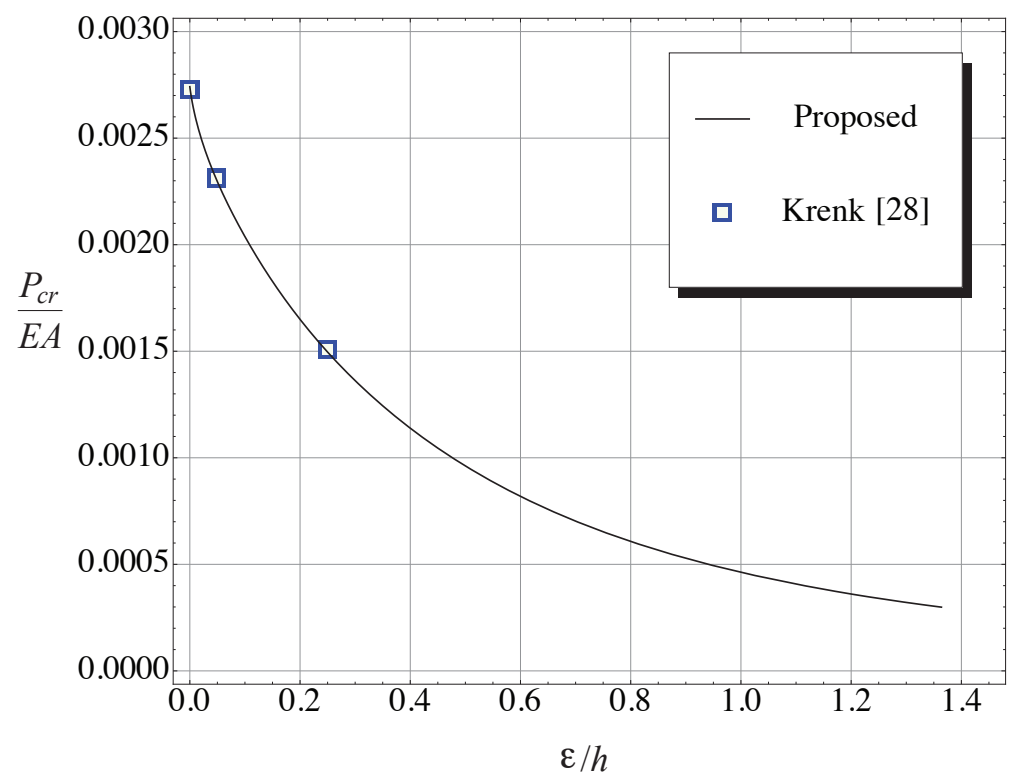

Figure 5: Stability boundary for the truss-spring system

\subsection{Four-bar 3D truss}

In this example, a 3D truss dome with complex behaviour (four-bar truss subjected to a vertical load $P$ at the top) is investigated (Figure 6). The width of the truss in the directions of axes $x$ and $z$ is $2 L(L=1000 \mathrm{~mm})$. $h$ represents the height of the dome. All elements have the same stiffness $(E A=1.0 \mathrm{kN})$. Depending on the value of $h$, the type of critical points varies. If $h<1224.72 \mathrm{~mm}$, the first critical point along the equilibrium path is a limit point. For higher magnitudes of $h$, there is a double bifurcation point before reaching the limit point. For $h=1224.72 \mathrm{~mm}$, a double bifurcation point and a limit point coincide at a critical load $3.578 \times 10^{-1} \mathrm{kN}$ [48].

A geometric control parameter (of magnitude $\varepsilon$ ) is considered for the top node in the direction $(1,1,1)$. The analysis starts from a critical state of the imperfect structure with $\varepsilon=-4 \mathrm{~mm}$ and corresponding critical load $3.547 \times 10^{-1} \mathrm{kN}$. Figure 7 illustrates the stability boundary for the four-bar truss. By comparing the critical points calculated by [48] and by the algorithm we propose, one can assess the performance of the proposed method. Table 2 provides a summary of 


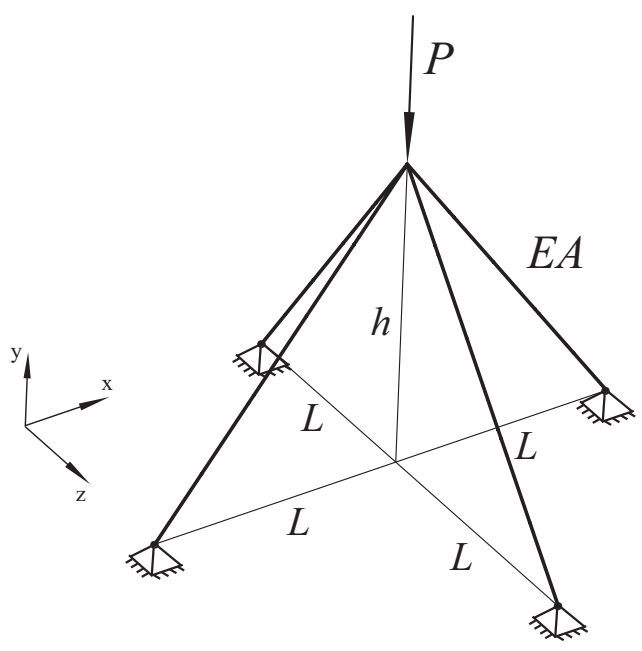

Figure 6: Four-bar 3D truss

computation obtained with $\Delta s=18.0$. Note that the procedure we introduced can track the stability boundary for any range of $\varepsilon$, while the perturbation method used in [48] is only valid for a limited domain of $\varepsilon$.

Table 2: Analysis of the four-bar 3D truss in Figure 6

\begin{tabular}{ccccccc}
\hline \multirow{2}{*}{ Increment } & No. of & \multicolumn{3}{c}{ Contribution of $\alpha_{i}$} & Control Par. & Critical load \\
\cline { 3 - 5 } & iterations & $\alpha_{1}\left(\mathrm{~mm}^{-1}\right)$ & $\alpha_{2}\left(\mathrm{kN}^{-1}\right)$ & $\alpha_{3}\left(\mathrm{~mm}^{-1}\right)$ & $\varepsilon(\mathrm{mm})$ & $P_{c r}(\mathrm{kN})$ \\
\hline 1 & 4 & $9.903 \times 10^{-1}$ & $9.919 \times 10^{-5}$ & $1.391 \times 10^{-1}$ & $-1.865 \times 10^{0}$ & $3.563 \times 10^{-1}$ \\
2 & 4 & $9.956 \times 10^{-1}$ & $7.071 \times 10^{-5}$ & $9.420 \times 10^{-2}$ & $-5.549 \times 10^{-1}$ & $3.573 \times 10^{-1}$ \\
3 & 4 & $9.987 \times 10^{-1}$ & $3.999 \times 10^{-5}$ & $5.054 \times 10^{-2}$ & $-1.957 \times 10^{-2}$ & $3.577 \times 10^{-1}$ \\
4 & 6 & $1.000 \times 10^{0}$ & $7.712 \times 10^{-6}$ & $9.266 \times 10^{-3}$ & $2.472 \times 10^{-1}$ & $3.577 \times 10^{-1}$ \\
5 & 3 & $9.995 \times 10^{-1}$ & $5.031 \times 10^{-6}$ & $3.111 \times 10^{-2}$ & $1.094 \times 10^{0}$ & $3.576 \times 10^{-1}$ \\
6 & 3 & $9.981 \times 10^{-1}$ & $1.125 \times 10^{-5}$ & $6.200 \times 10^{-2}$ & $2.461 \times 10^{0}$ & $3.573 \times 10^{-1}$ \\
7 & 3 & $9.961 \times 10^{-1}$ & $1.768 \times 10^{-5}$ & $8.845 \times 10^{-2}$ & $4.280 \times 10^{0}$ & $3.569 \times 10^{-1}$ \\
\hline
\end{tabular}

Table 3: Convergence of the proposed method at the fourth increment (four-bar truss)

\begin{tabular}{ccc}
\hline Iteration & $\left|\delta \mathbf{u}_{i}{ }^{T} \mathbf{r}_{i}\right|(\mathrm{kN.mm})$ & $\left|\lambda_{i}\right|(\mathrm{kN} / \mathrm{mm})$ \\
\hline 1 & $3.24450 \times 10^{-3}$ & $1.924 \times 10^{-5}$ \\
2 & $1.74961 \times 10^{-3}$ & $5.248 \times 10^{-6}$ \\
3 & $1.16368 \times 10^{-5}$ & $5.526 \times 10^{-7}$ \\
4 & $7.09990 \times 10^{-10}$ & $4.171 \times 10^{-9}$ \\
5 & $2.49799 \times 10^{-18}$ & $2.506 \times 10^{-13}$ \\
6 & $3.24166 \times 10^{-35}$ & $9.012 \times 10^{-22}$ \\
\hline
\end{tabular}




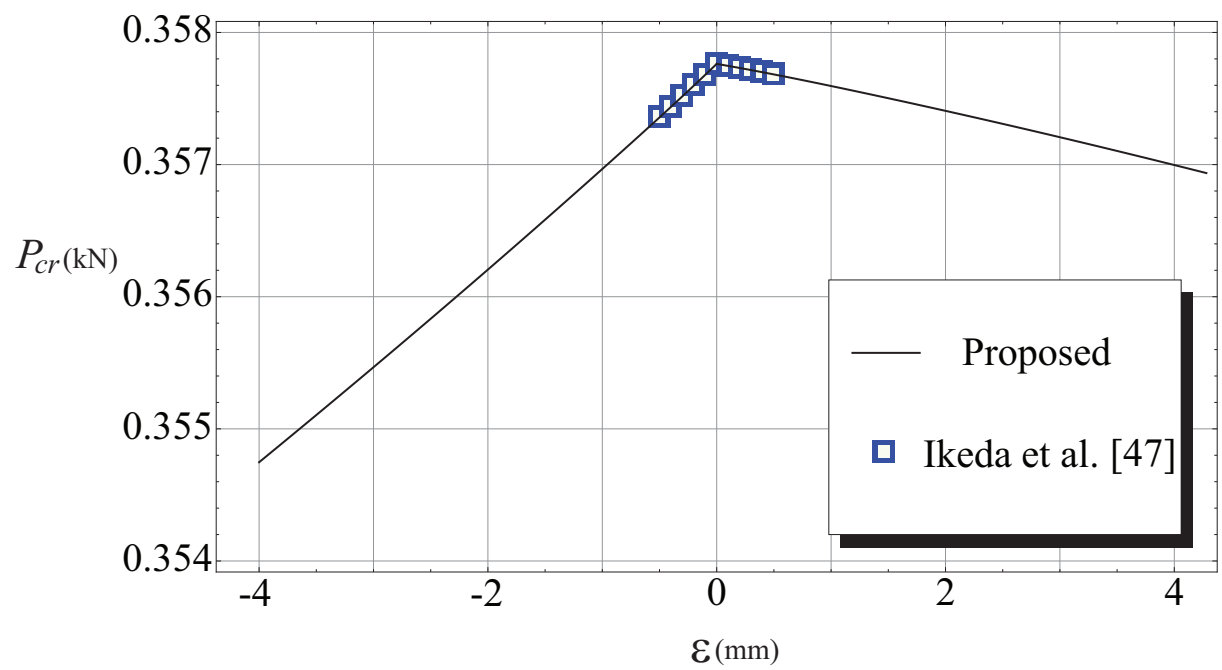

Figure 7: Stability boundary for a four-bar 3D truss

Along the stability boundary, the nature of the critical point changes. For cases of $\varepsilon<0 \mathrm{~mm}$ and $\varepsilon>0 \mathrm{~mm}$, we observe limit and simple bifurcation points, respectively. In addition, there is a double bifurcation point at the hilltop point $(\varepsilon=0 \mathrm{~mm})$ on the stability boundary [48]. The proposed incrementaliterative method can successfully pass this point by using two eigenvectors of the repeated null eigenvalue at the corresponding (fourth) increment. Table 3 shows the quadratic convergence of the proposed method at this increment. For all other incremental and iterative steps, since there is a single zero eigenvalue on the stability boundary, only one eigenvector is computed and used.

\subsection{Plane truss arch}

Figure 8 illustrates a 2D truss arch subjected to vertical loads at the top nodes. This structure has 35 bar-members and 34 degrees of freedom. All members have the same stiffness $\left(E A=1.0 \times 10^{7} \mathrm{~N}\right)$. The nodal coordinates are given in Table 4 .

The main external load vector is applied to half of the structure; its magnitude is a function of the load parameter $P$. For this case $P_{c r}=7.771 \times 10^{1} \mathrm{~N}$. In this example, the additional parameter is a secondary load vector in terms of 


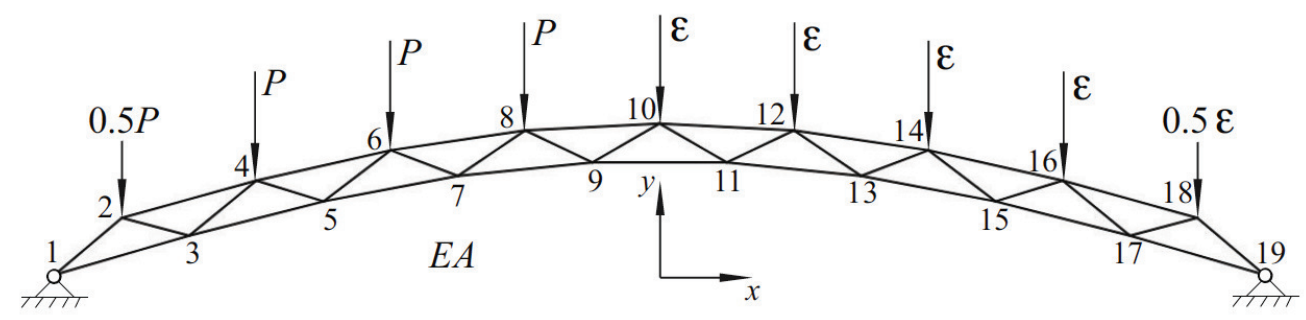

Figure 8: Plane truss arch

Table 4: Coordinates of nodes for the plane truss arch

\begin{tabular}{ccc}
\hline Nodal No. & x coordinates $(\mathrm{cm})$ & y coordinates $(\mathrm{cm})$ \\
\hline 1,19 & $\mp 342.9$ & 0.0 \\
2,18 & $\mp 304.8$ & 5.065 \\
3,17 & $\mp 266.7$ & 3.475 \\
4,16 & $\mp 228.6$ & 8.382 \\
5,15 & $\mp 190.5$ & 6.530 \\
6,14 & $\mp 152.4$ & 11.085 \\
7,13 & $\mp 114.3$ & 8.799 \\
8,12 & $\mp 76.2$ & 12.850 \\
9,11 & $\mp 38.1$ & 10.005 \\
10 & 0.0 & 13.462 \\
\hline
\end{tabular}

$\varepsilon$. The proposed algorithm (with $\Delta s=9.5$ ) is used to trace the stability boundary, which is composed of two curves connected by a cusp point (Figure 9). All critical points forming the stability boundary are limit points. Although the shape of the buckling mode changes at this point, the proposed procedure is able to pass from one side of the cusp to the other. The summary of computations is given in Table 5. Figure 9 shows that there can be more than one critical load for some values of $\varepsilon$. The loading pattern of the main and the additional load may result in different critical values. For example, there are three values for the critical load corresponding to $\varepsilon=58 \mathrm{~N}$, and they can be obtained from loading patterns $\mathbf{A}, \mathbf{B}$ and $\mathbf{C}$ (Figure 9 ). In the pattern $\mathbf{A}$, the secondary load parameter $\varepsilon$ increases from the unloaded state to $58 \mathrm{~N}$, then, the main external load vector is applied until the truss reaches its stability boundary. The sequence of loadings is vice versa in the $\mathbf{B}$ pattern. In $\mathbf{C}$, the ratio between the load parameters $P$ and $\varepsilon$ is constant during loading. Figure 10 shows the deformed 
shape of the truss at these critical states. Note that, the critical points given by [26] are consistent with the stability boundary calculated by the proposed method. Moreover, our method can handle multiple bifurcation points, whereas the method in [26] cannot.

Table 5: Analysis of the plane truss arch from Figure 8

\begin{tabular}{|c|c|c|c|c|c|c|}
\hline \multirow{2}{*}{ Increment } & \multirow{2}{*}{$\begin{array}{l}\text { No. of } \\
\text { iterations }\end{array}$} & \multicolumn{3}{|c|}{ Contribution of $\alpha_{i}$} & \multirow{2}{*}{$\begin{array}{c}\text { Control Par. } \\
\varepsilon(\mathrm{N})\end{array}$} & \multirow{2}{*}{$\begin{array}{c}\text { Critical load } \\
P_{c r}(\mathrm{~N})\end{array}$} \\
\hline & & $\alpha_{1}\left(\mathrm{~cm}^{-1}\right)$ & $\alpha_{2}\left(\mathrm{~N}^{-1}\right)$ & $\alpha_{3}\left(\mathrm{~N}^{-1}\right)$ & & \\
\hline 1 & 4 & $1.423 \times 10^{-1}$ & $5.038 \times 10^{-1}$ & $8.520 \times 10^{-1}$ & $1.059 \times 10^{1}$ & $7.184 \times 10^{1}$ \\
\hline 2 & 4 & $1.428 \times 10^{-1}$ & $4.537 \times 10^{-1}$ & $8.796 \times 10^{-1}$ & $2.108 \times 10^{1}$ & $6.686 \times 10^{1}$ \\
\hline 3 & 4 & $1.478 \times 10^{-1}$ & $3.919 \times 10^{-1}$ & $9.081 \times 10^{-1}$ & $3.140 \times 10^{1}$ & $6.291 \times 10^{1}$ \\
\hline 4 & 4 & $1.595 \times 10^{-1}$ & $3.111 \times 10^{-1}$ & $9.369 \times 10^{-1}$ & $4.149 \times 10^{1}$ & $6.017 \times 10^{1}$ \\
\hline 5 & 5 & $1.840 \times 10^{-1}$ & $1.960 \times 10^{-1}$ & $9.632 \times 10^{-1}$ & $5.134 \times 10^{1}$ & $5.904 \times 10^{1}$ \\
\hline 6 & 7 & $2.438 \times 10^{-1}$ & $5.148 \times 10^{-3}$ & $9.698 \times 10^{-1}$ & $6.108 \times 10^{1}$ & $6.114 \times 10^{1}$ \\
\hline 7 & 6 & $7.893 \times 10^{-1}$ & $3.566 \times 10^{-1}$ & $4.999 \times 10^{-1}$ & $5.676 \times 10^{1}$ & $4.143 \times 10^{1}$ \\
\hline 8 & 4 & $1.643 \times 10^{-1}$ & $9.853 \times 10^{-1}$ & $4.660 \times 10^{-2}$ & $5.671 \times 10^{1}$ & $3.179 \times 10^{1}$ \\
\hline 9 & 4 & $1.443 \times 10^{-1}$ & $9.890 \times 10^{-1}$ & $3.177 \times 10^{-2}$ & $5.732 \times 10^{1}$ & $2.219 \times 10^{1}$ \\
\hline 10 & 4 & $1.346 \times 10^{-1}$ & $9.868 \times 10^{-1}$ & $8.972 \times 10^{-2}$ & $5.843 \times 10^{1}$ & $1.256 \times 10^{1}$ \\
\hline 11 & 4 & $1.299 \times 10^{-1}$ & $9.821 \times 10^{-1}$ & $1.364 \times 10^{-1}$ & $5.997 \times 10^{1}$ & $2.895 \times 10^{0}$ \\
\hline
\end{tabular}

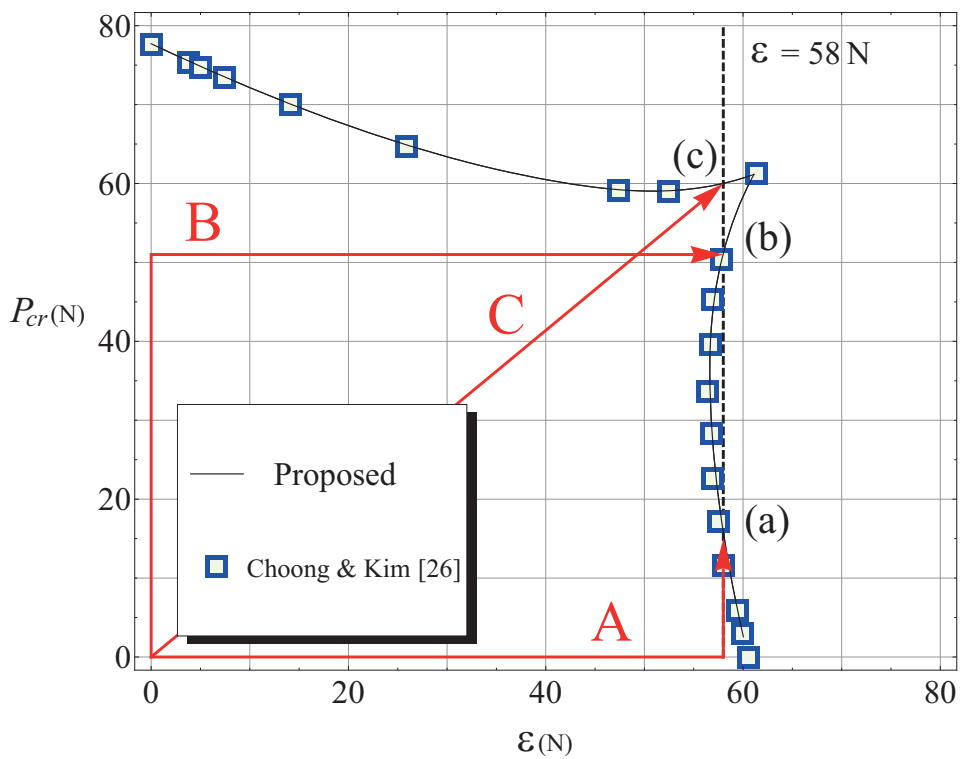

Figure 9: Stability boundary for the plane truss arch

In order to show the relationship between the time of the analysis and the 


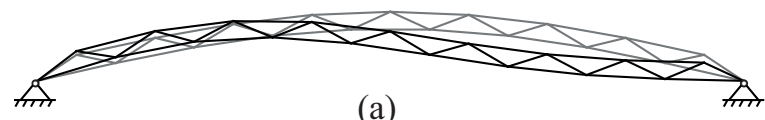

(a)

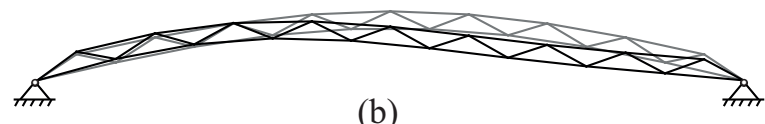

(b)

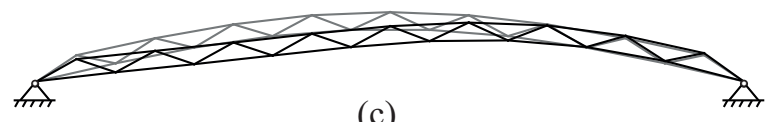

(c)

Figure 10: Deformed shape at critical states corresponding to loading patterns: (a) A (b) B (c) $\mathbf{C}$

number of degrees of freedom (DoFs), we generate a 3D truss based on the scheme of the 2D truss in Figure 8. Two, four, eight and sixteen rows of the plane truss are placed parallel at equal distance $(h=7.62 \mathrm{~cm})$ and linked transversally by truss-bar elements with the same stiffness $\left(E A=1.0 \times 10^{7} \mathrm{~N}\right)$. Figure 11 (a)-(d) illustrates the unloaded state of these trusses. The number of DoFs is 104, 210, 422 and 846 for the two, four, eight and sixteen-rows 3D truss, respectively. If the loading scheme in Figure 8 is applied on each row, we observe the same behaviour for the stability boundary for all trusses. Figure $11(\mathrm{e})-(\mathrm{h})$ shows the deformed shapes of 3D trusses at their critical load for $\varepsilon=0 \mathrm{~N}$.

One can assume that the relationship between the time of the analysis and the number of DoFs is

$$
(\text { Time })=\alpha(\mathrm{DoF})^{\beta} \text {. }
$$

In this equation, the coefficient $\alpha$ is a function of the computer configuration and other parameters which are not of interest here. The coefficient $\beta$ represents the increase rate in the number of operations due to the increase in the number of DoFs. Eq. (52) can be rewritten

$$
\log (\text { Time })=(\log \alpha)+\beta \log (\mathrm{DoF})
$$

where $\beta$ is the slope of the line in the logarithmic scale. 


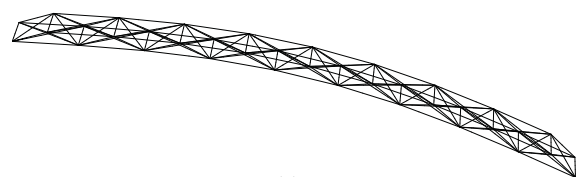

(a)

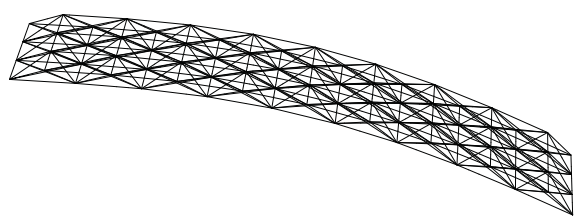

(b)

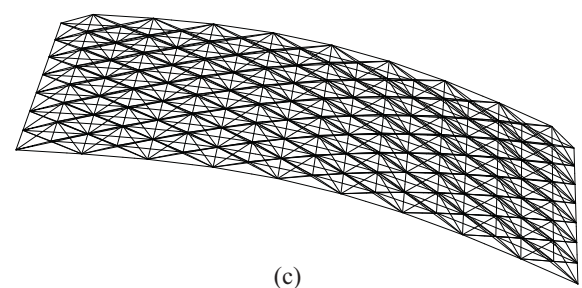

(c)

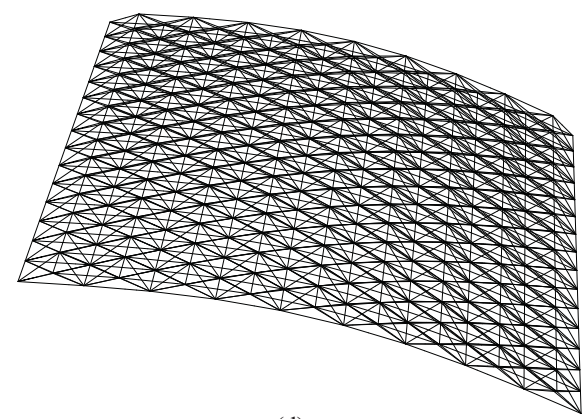

(d)

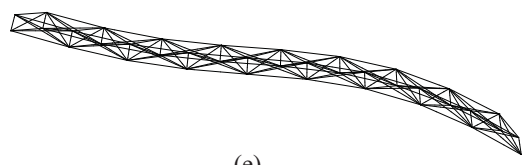

(e)

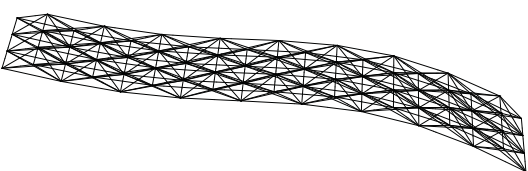

(f)

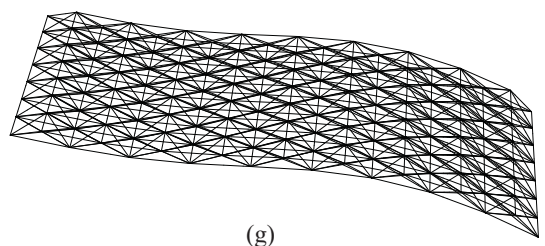

(g)

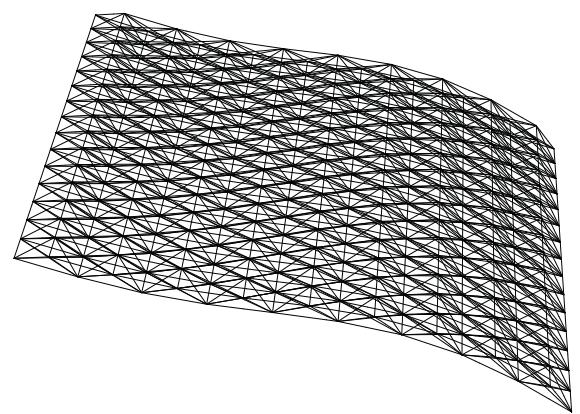

(h)

Figure 11: Two, four, eight and sixteen-rows 3D trusses: (a)-(d) unloaded state, (e)-(h) deformed shape at the critical load

Three types of analyses are performed for all 3D trusses and the results are shown in Figure 12. First, the cylindrical arc-length procedure is applied for tracing the equilibrium path for 60 incremental and iterative steps. The slope 
of the regression line is $\beta_{1}=2.45$. The second analysis is the equilibrium path following by the cylindrical arc-length method accompanied by a critical point test function. Here, the test function is the smallest eigenvalue computed by Rayleigh quotient iteration at the begining of each increment. The slope of the regression line for this analysis is $\beta_{2}=2.86$. Tracing the stability boundary by the proposed method is the third analysis. In order to maintain a quadratic convergence behaviour, the smallest eigenvalue and the corresponding eigenvector are computed and used in all increments and iterations. The slope of the regression line is close to the other analyses $\left(\beta_{3}=2.81\right)$. Therefore, the increase rate in the cost of the proposed method for structures with higher DoFs is appropriate and comparable with Newton-like methods used for computing the equilibrium path.

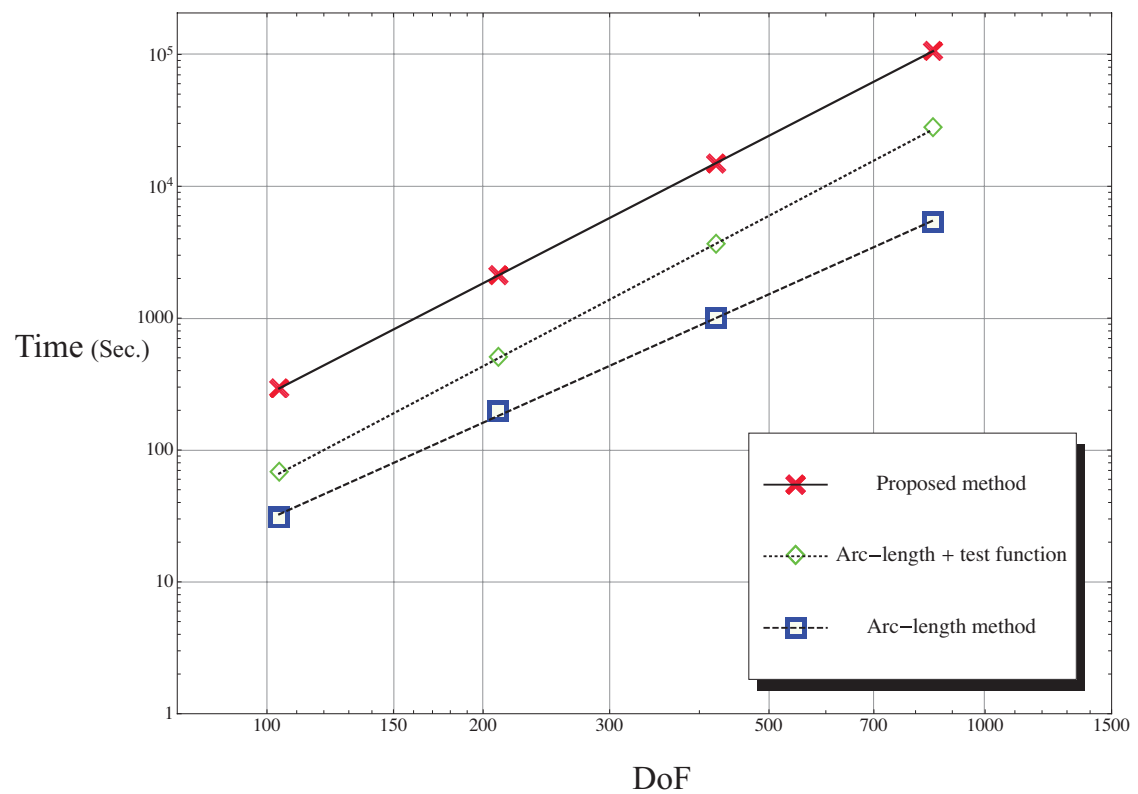

Figure 12: Relationship between the time of the analysis and the number of DoFs

\subsection{Shallow arch frame}

In this example, the effect of temperature variation on the critical load is investigated for a set of circular hallow arches with different radii. Figure 13 
shows the material properties and the geometry of the system. The geometry is defined by: the distance between the two supports $(L)$, the radius of curvature $(R)$, the thickness $(t)$, and the transversal depth $(d)$. For this analysis, the finite element method is used. We use 20 Timoshenko beam elements with large transverse displacement to discretize the arch. The beam is subjected to a vertical load at the top point. The control parameter $\varepsilon$ is the temperature change:

$$
\varepsilon=\Delta T=T-T_{r e f},
$$

where $T$ is the absolute temperature measured in Kelvin. $T_{r e f}$ is defined as the zero-stress reference temperature. Here, $T_{r e f}=300 \mathrm{~K}$. For one-dimensional elements with the thermal expansion coefficient $\alpha$, the thermal stress can be calculated $[2,16]$ :

$$
\sigma=-E \alpha \Delta T
$$

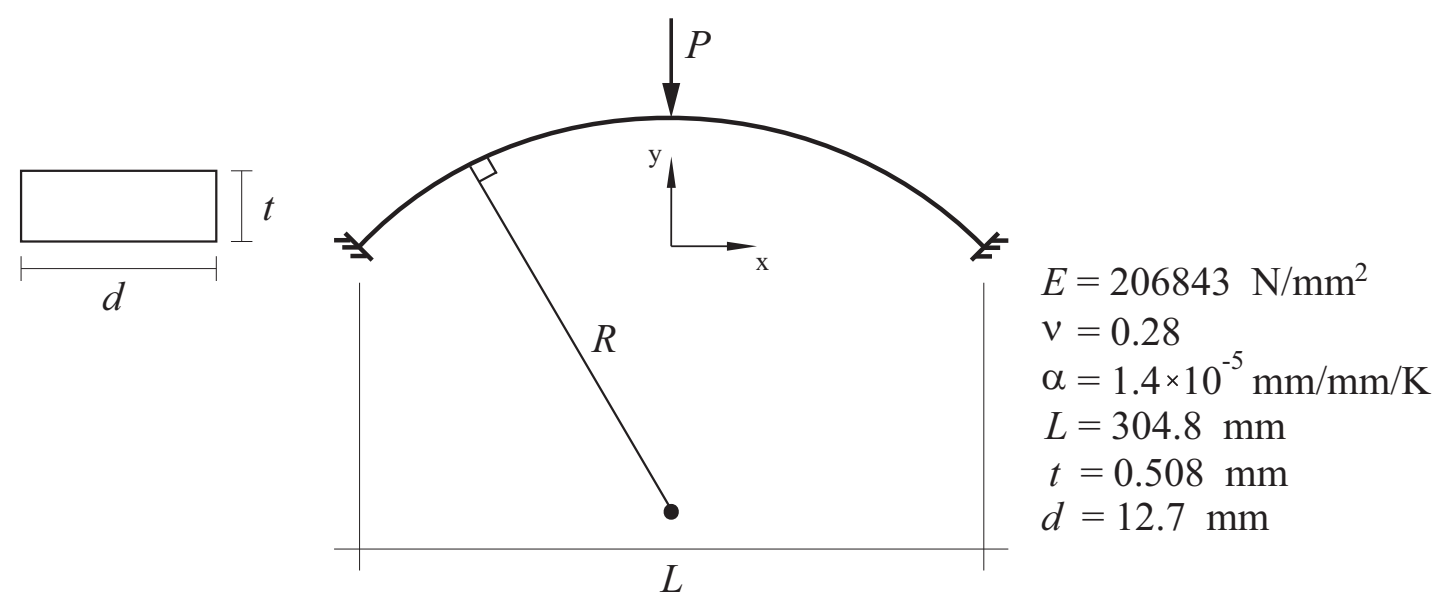

Figure 13: Shallow arch frame

The proposed method is applied using $\Delta s=2.5$ to seven shallow arches. Table 6 shows the average number of iterations per increment during the identification of the stability boundary for all arches. The analyses start from the critical state at $\Delta T=+20 \mathrm{~K}$. Figure 14 illustrates the stability boundaries. 
Note that the critical points calculated indirectly in [49] match the results obtained with the new method.

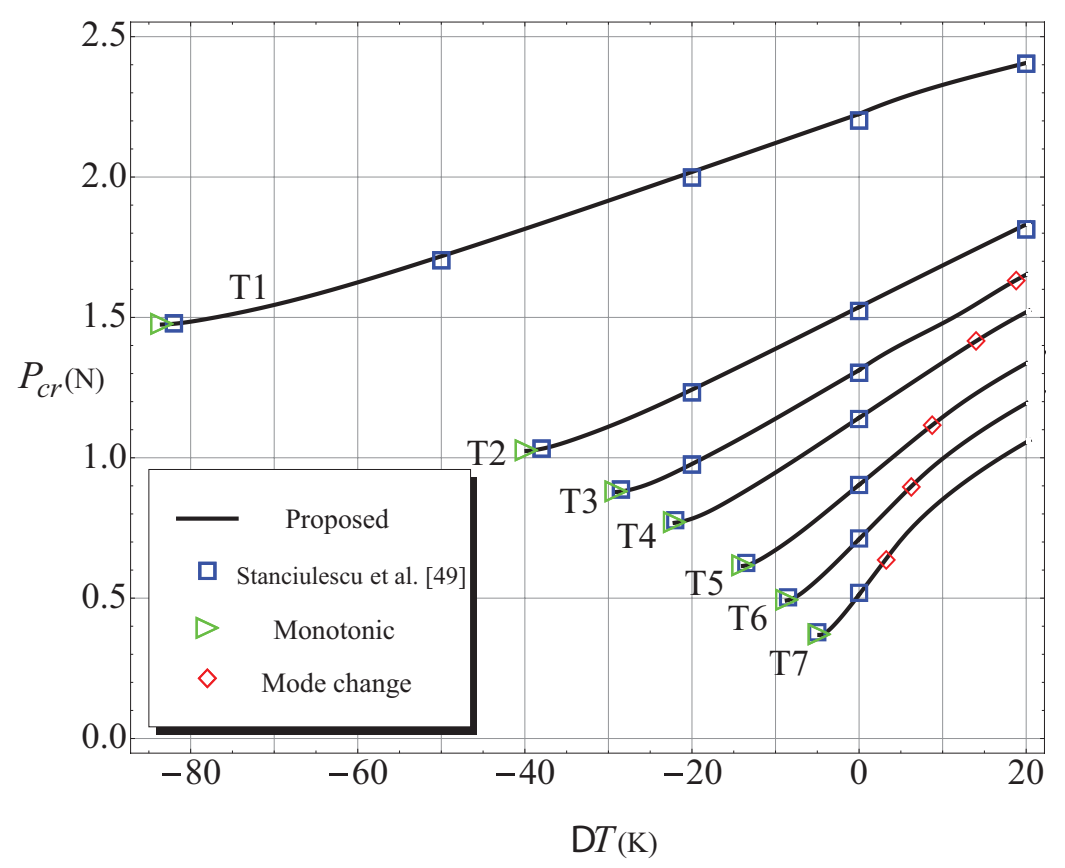

Figure 14: Stability boundaries for the shallow arch frame with different radii

Table 6: Analysis of the shallow arch frame

\begin{tabular}{ccc}
\hline Name & $R(\mathrm{~mm})$ & $\begin{array}{c}\text { Average No. of iterations } \\
\text { per increment }\end{array}$ \\
\hline T1 & 1270.0 & 4.025 \\
T2 & 1828.8 & 4.043 \\
T3 & 2133.6 & 4.105 \\
T4 & 2438.4 & 5.063 \\
T5 & 3048.0 & 4.231 \\
T6 & 3810.0 & 4.636 \\
T7 & 5080.0 & 4.333 \\
\hline
\end{tabular}

For $\Delta T=0 \mathrm{~K}$, there are two limit points on the equilibrium path. By decreasing the temperature, the limit points get closer to each other. Figure 15 displays changes in the equilibrium path due to variation in $\varepsilon$ for the arch T2. For a specific $\varepsilon$, the limit points join each other. The primary equilibrium path 
in this state can be named monotonic [49]. In case of lower temperature, there is no critical point on the equilibrium path. In Figure 14, the proposed procedure has been stopped when it reached the minimum value of $\varepsilon$ (triangles). If our interest is to let the procedure continue after reaching the monotonic state, it will switch to the stability boundary corresponding to the second limit point. Figure 16 shows the stability boundaries of T2. The solid and dashed lines belong to the first and the second limit points, respectively. These boundaries divide the plane $p$ and $\varepsilon$ into two areas $\mathbf{A}$ and $\mathbf{B}$. For fixed values of $p$ and $\varepsilon$ in $\mathbf{B}$, the minimum number of solutions for $\mathbf{u}$ is equal to one, while a point in $\mathbf{A}$ area gives at least three solutions.

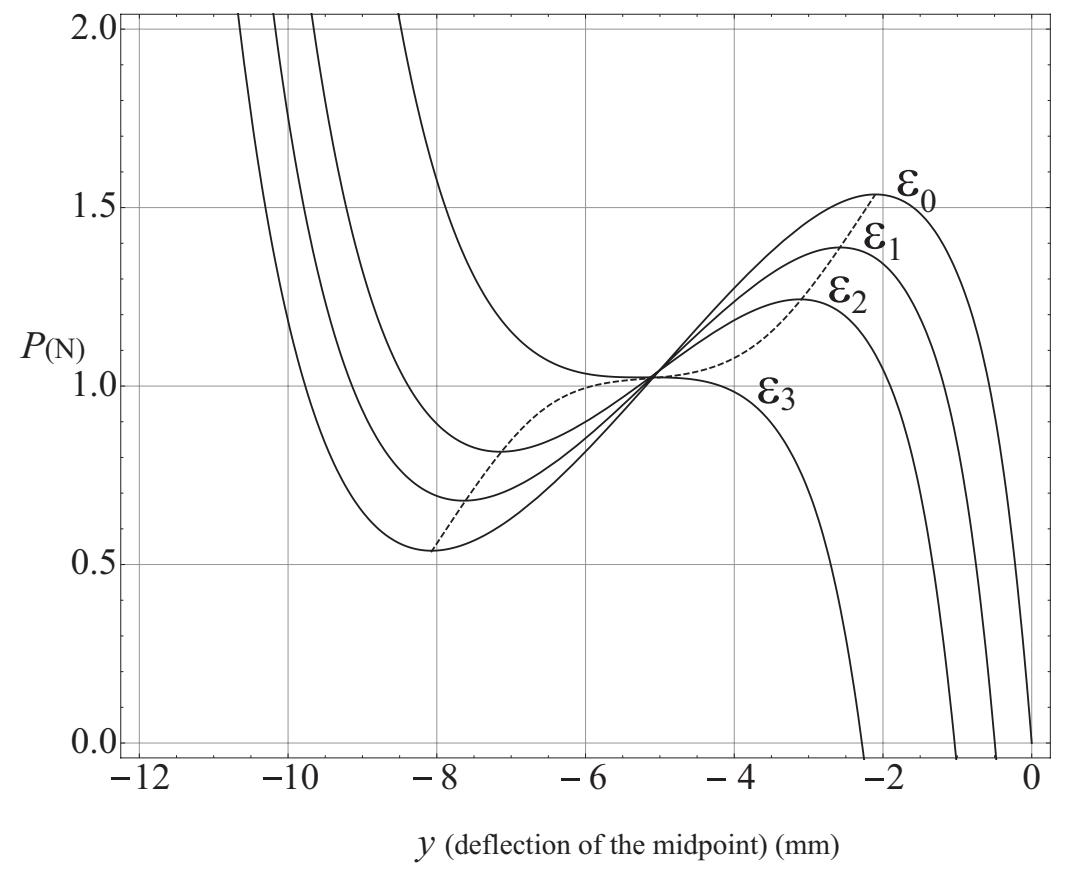

Figure 15: Equilibrium paths for the beam T2 $\left(\varepsilon_{3}<\varepsilon_{2}<\varepsilon_{1}<\varepsilon_{0}\right)$

For $\Delta T>0 \mathrm{~K}$, one can observe a change in buckling mode from symmetric to asymmetric. Actually, by increasing the temperature, a bifurcation point determines the magnitude of the critical load. Diamonds in Figure 14 identify the position of this mode change on stability boundaries. The proposed algorithm 


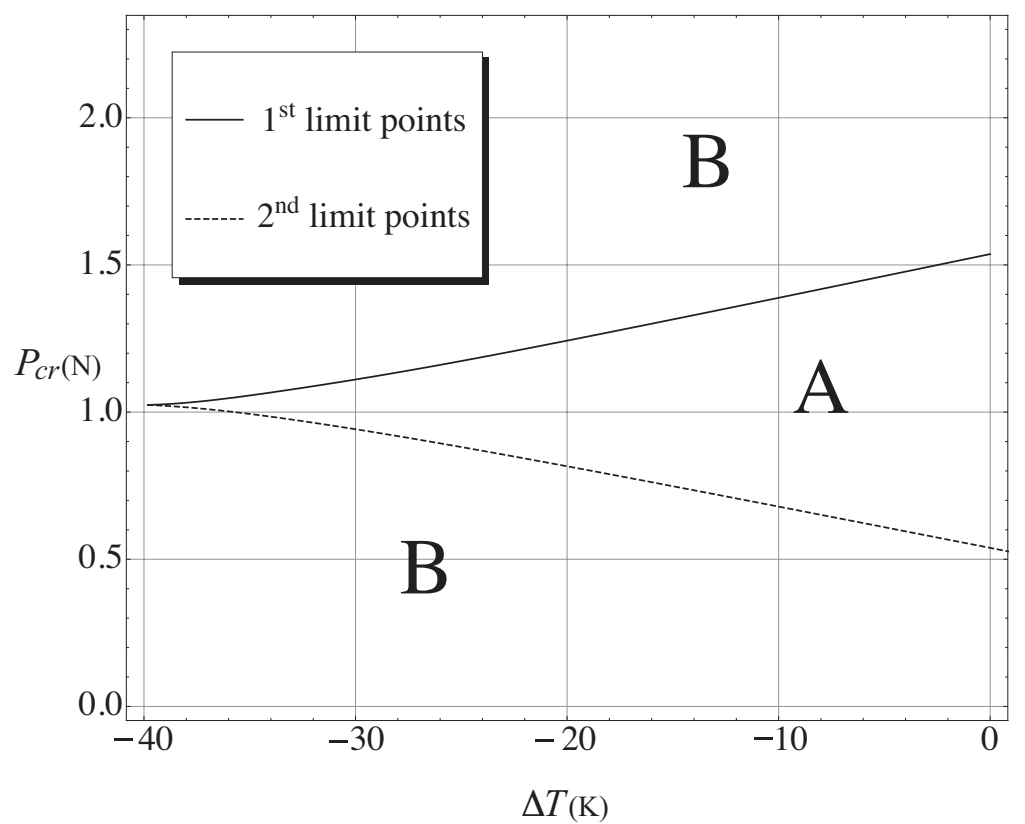

Figure 16: Stability boundaries for the beam T2

is able to pass these points successfully.

\subsection{Cylindrical shell}

Here, the proposed method is tested on a shell structure often discussed in the literature $[41,50,51]$. The structure is a cylindrical shell and is subjected to a central point load (Figure 17). The longitudinal edges are hinged, while the curved edges are free. The material and geometrical properties of the shell are given in Figure 17.

The thickness of the shell $(t)$ is the parameter that can influence the loadbearing capacity of the structure [39, 52]. $16 \times 16$ shell elements (with four nodes and 20 degrees of freedom) are used for the spatial discretization (with a total of 1343 DoFs). The initial thickness is $t_{0}=4.35 \mathrm{~mm}$, and $P_{c r}\left(t_{0}\right)=$ $0.19062 \mathrm{kN}$. In this example, the thickness of the cylindrical shell is a function of a control parameter $t(\varepsilon)=t_{0}+\varepsilon$. Figure 18 illustrates the stability boundaries of the structure. 


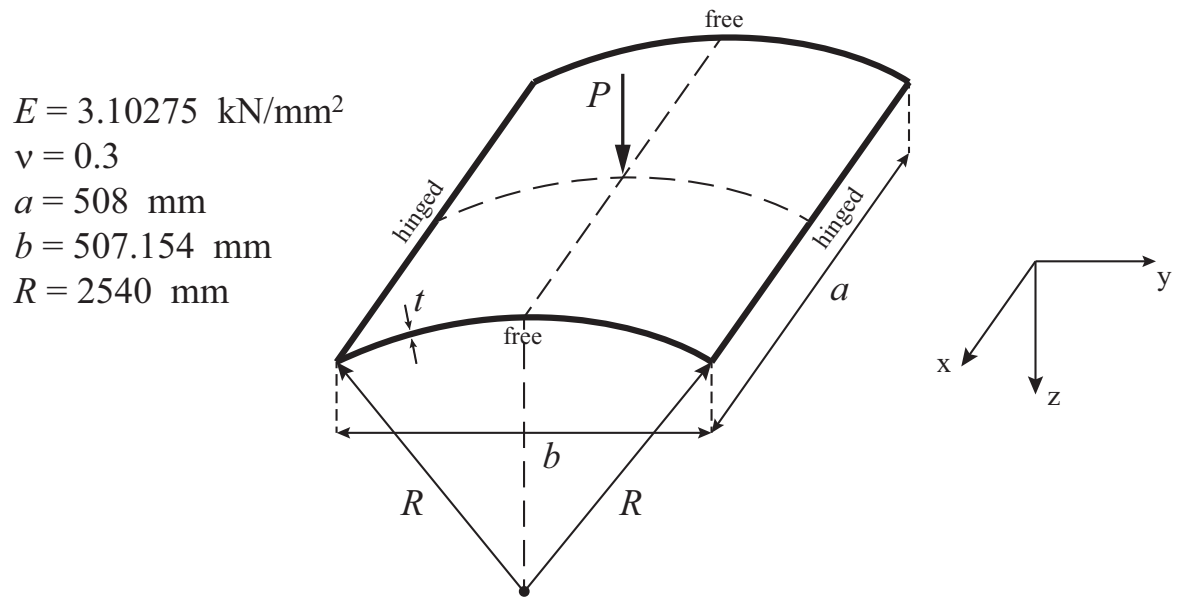

Figure 17: Cylindrical shell

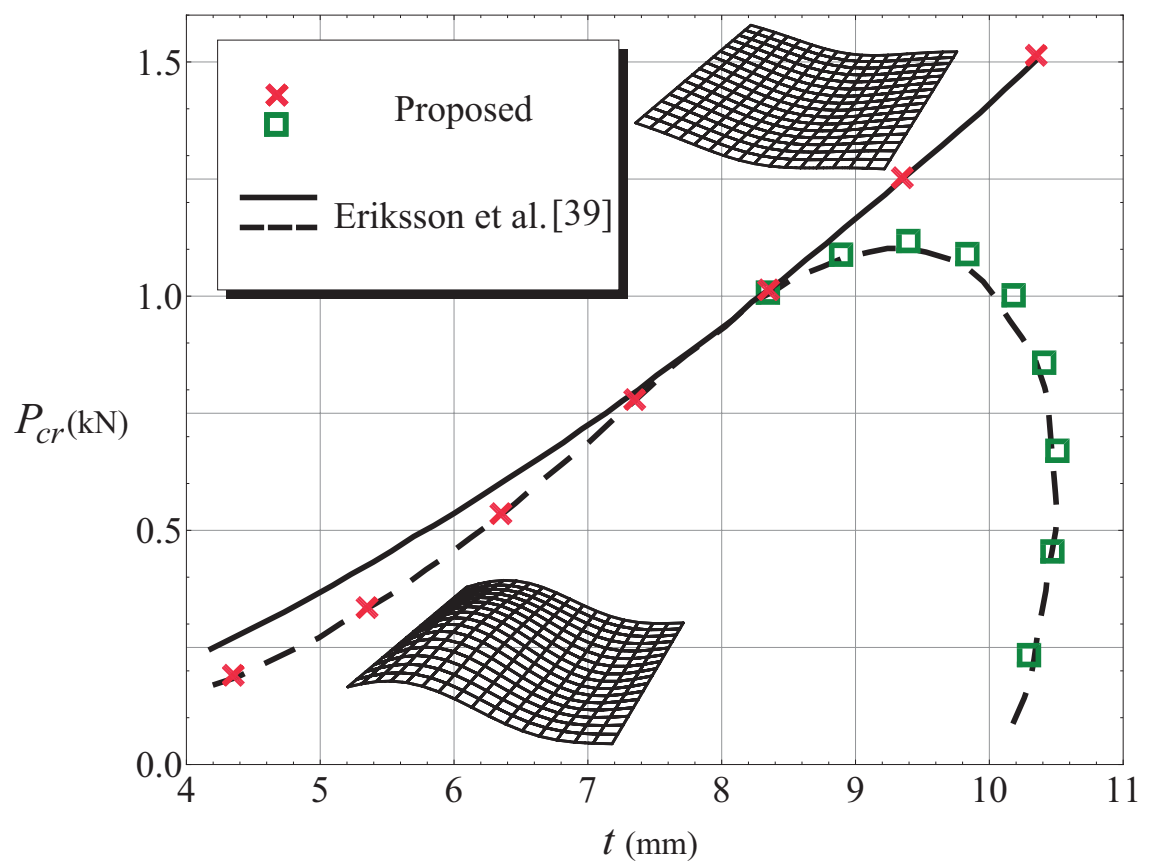

Figure 18: Stability boundaries for the cylindrical shell

In this figure, the crosses show the relationship between the critical load and the thickness computed by the proposed method. In order to obtain $P_{c r}$ relative to regular values of thickness $(t=4.35,5.35, \ldots, 10.35)$, the parameters $\alpha_{1}, \alpha_{2}$ 
and $\alpha_{3}$ are set to $0 \mathrm{~mm}^{-1}, 0 \mathrm{kN}^{-1}$ and $1 \mathrm{~mm}^{-1}$, respectively. Consequently, based on Eq. (44), $\alpha_{3} \Delta \varepsilon=\Delta s=1.0$ throughout the analysis. Table 7 provides the magnitudes of the residual energy $\left(\delta \mathbf{u}_{i}{ }^{T} \mathbf{r}_{i}\right)$, the smallest eigenvalue $\left(\lambda_{i}\right)$, variations in the load parameter $\left(\delta p_{i}\right)$ and the computed critical load $\left(P_{c r}\right)$ along the analysis, and demonstrates the fast convergence of the proposed method.

Table 7: Analysis of the cylindrical shell

\begin{tabular}{|c|c|c|c|c|c|}
\hline$t(\mathrm{~mm})$ & Inc. - Ite. & $\left|\delta \mathbf{u}_{i}{ }^{T} \mathbf{r}_{i}\right|$ (kN.mm) & $\left|\lambda_{i}\right|(\mathrm{kN} / \mathrm{mm})$ & $\delta p_{i}(\mathrm{kN})$ & $P_{c r}(\mathrm{kN})$ \\
\hline \multirow{5}{*}{5.35} & $1-0$ & $4.8491 \times 10^{0}$ & $4.9557 \times 10^{-1}$ & $1.1775 \times 10^{-1}$ & 0.30837 \\
\hline & $1-1$ & $1.3400 \times 10^{0}$ & $3.6510 \times 10^{-4}$ & $2.7025 \times 10^{-2}$ & 0.33540 \\
\hline & $1-2$ & $5.0064 \times 10^{-5}$ & $3.0616 \times 10^{-7}$ & $-3.2517 \times 10^{-4}$ & 0.33507 \\
\hline & $1-3$ & $2.5988 \times 10^{-10}$ & $5.3303 \times 10^{-14}$ & $7.9037 \times 10^{-8}$ & 0.33507 \\
\hline & $1-4$ & $2.4779 \times 10^{-17}$ & $1.7265 \times 10^{-26}$ & $7.8008 \times 10^{-12}$ & 0.33507 \\
\hline \multirow{5}{*}{6.35} & $2-0$ & $7.6043 \times 10^{0}$ & $4.9696 \times 10^{-1}$ & $1.7237 \times 10^{-1}$ & 0.50744 \\
\hline & $2-1$ & $5.8037 \times 10^{-1}$ & $2.1850 \times 10^{-3}$ & $2.8863 \times 10^{-2}$ & 0.53630 \\
\hline & $2-2$ & $6.2136 \times 10^{-5}$ & $9.8338 \times 10^{-7}$ & $-7.0693 \times 10^{-4}$ & 0.53559 \\
\hline & $2-3$ & $1.5603 \times 10^{-9}$ & $3.3394 \times 10^{-11}$ & $2.2523 \times 10^{-7}$ & 0.53559 \\
\hline & $2-4$ & $1.0286 \times 10^{-18}$ & $2.3846 \times 10^{-21}$ & $9.3867 \times 10^{-14}$ & 0.53559 \\
\hline \multirow{5}{*}{7.35} & $3-0$ & $4.1790 \times 10^{0}$ & $1.7628 \times 10^{-1}$ & $2.2703 \times 10^{-1}$ & 0.76262 \\
\hline & $3-1$ & $3.2620 \times 10^{0}$ & $1.9332 \times 10^{-3}$ & $1.8087 \times 10^{-2}$ & 0.78071 \\
\hline & $3-2$ & $6.1558 \times 10^{-4}$ & $2.4604 \times 10^{-6}$ & $-1.5441 \times 10^{-3}$ & 0.77916 \\
\hline & $3-3$ & $2.7359 \times 10^{-8}$ & $2.1690 \times 10^{-12}$ & $7.7786 \times 10^{-7}$ & 0.77916 \\
\hline & $3-4$ & $1.8878 \times 10^{-18}$ & $1.4192 \times 10^{-21}$ & $-2.7758 \times 10^{-12}$ & 0.77916 \\
\hline \multirow{5}{*}{8.35} & $4-0$ & $1.9691 \times 10^{1}$ & $6.3383 \times 10^{-2}$ & $2.5047 \times 10^{-1}$ & 1.02963 \\
\hline & $4-1$ & $4.6475 \times 10^{0}$ & $1.9094 \times 10^{-3}$ & $-1.5686 \times 10^{-2}$ & 1.01395 \\
\hline & $4-2$ & $8.1451 \times 10^{-2}$ & $1.4237 \times 10^{-5}$ & $-5.8864 \times 10^{-4}$ & 1.01336 \\
\hline & $4-3$ & $1.3389 \times 10^{-6}$ & $1.2487 \times 10^{-10}$ & $-1.2654 \times 10^{-5}$ & 1.01335 \\
\hline & $4-4$ & $1.4099 \times 10^{-15}$ & $1.3204 \times 10^{-18}$ & $-5.6032 \times 10^{-11}$ & 1.01335 \\
\hline \multirow{5}{*}{9.35} & $5-0$ & $5.4949 \times 10^{0}$ & $6.0936 \times 10^{-3}$ & $2.2825 \times 10^{-1}$ & 1.24160 \\
\hline & $5-1$ & $1.2405 \times 10^{0}$ & $7.4211 \times 10^{-5}$ & $1.1251 \times 10^{-2}$ & 1.25285 \\
\hline & $5-2$ & $5.9169 \times 10^{-5}$ & $1.4760 \times 10^{-9}$ & $-6.8814 \times 10^{-5}$ & 1.25278 \\
\hline & $5-3$ & $2.4282 \times 10^{-13}$ & $2.1448 \times 10^{-17}$ & $7.2613 \times 10^{-10}$ & 1.25278 \\
\hline & $5-4$ & $1.3544 \times 10^{-29}$ & $9.0401 \times 10^{-34}$ & $4.1949 \times 10^{-18}$ & 1.25278 \\
\hline \multirow{5}{*}{10.35} & $6-0$ & $5.5918 \times 10^{0}$ & $4.6084 \times 10^{-3}$ & $2.5050 \times 10^{-1}$ & 1.50328 \\
\hline & $6-1$ & $8.0985 \times 10^{-1}$ & $6.2166 \times 10^{-5}$ & $1.0960 \times 10^{-2}$ & 1.51424 \\
\hline & $6-2$ & $1.6859 \times 10^{-5}$ & $5.8667 \times 10^{-10}$ & $-2.7148 \times 10^{-5}$ & 1.51421 \\
\hline & $6-3$ & $5.6160 \times 10^{-15}$ & $4.0745 \times 10^{-19}$ & $-1.2079 \times 10^{-10}$ & 1.51421 \\
\hline & $6-4$ & $3.7659 \times 10^{-33}$ & $2.0916 \times 10^{-37}$ & $-8.0685 \times 10^{-20}$ & 1.51421 \\
\hline
\end{tabular}

The variation in the thickness can change the stability behaviour of the cylindrical shell $[39,52]$. For $t<8 \mathrm{~mm}$, during an analysis that starts at the 
unloaded state, the first critical point encountered on the equilibrium path is a bifurcation point, while for $t>8 \mathrm{~mm}$, a limit point appears before the bifurcation. The proposed procedure automatically traces the stability boundary corresponding to the first critical point (the only critical point that the structure can reach following a stable equilibrium path), and successfully switches from bifurcation points to the locus of limit points when $t$ passes $8 \mathrm{~mm}$. Figure 18 shows this branch switching. The critical modes corresponding to the bifurcation and limit points are illustrated in this figure.

The proposed method can also trace the stability boundaries relative to the second or further critical points on the equilibrium path (which belong to unstable portions of the path). This goal can be achieved when the eigenvector corresponding to the former critical modes are omitted from $\boldsymbol{\Psi}_{i}$ in Eq. (36). By doing this, the method traces the stability boundary corresponding to bifurcation points for $t>8 \mathrm{~mm}$ (squares in Figure 18). For this part of analysis, $\Delta s=10.0$, and Eq. (50) specifies the contributions of $\Delta \mathbf{u}, \Delta p$ and $\Delta \varepsilon$ in the arc-length constraint (44). As it can be seen, the computed stability boundaries are in good agreement with the results obtained by [39]. Here, the solid and dashed lines represent the locus of limit and bifurcation points, respectively.

\section{Conclusions}

The load-bearing capacity of structures is usually sensitive to variations (imperfections) in geometrical, mechanical and loading parameters. Consequently, finding a relationship between the critical load and the magnitude of control parameters provides a better understanding of the structural behaviour. In this paper, a powerful method for tracking the stability boundary (without tracing the equilibrium path) for conservative systems is developed. In this method, each critical point, which is relative to a specific control parameter, can be directly obtained from the previously calculated critical point.

The proposed technique introduces a new incremental-iterative procedure to find various critical points (such as, limit, simple and multiple bifurcation) lying on the stability boundary. It is also capable to trace the branches of bifurcated 
stability boundaries. The system of equilibrium equations supplemented with a criticality condition and a spherical arc-length constraint is solved via Newton's method. The authors also suggest a formula to update the spherical constraint in each increment to improve convergence.

The numerical examples show the effectiveness of the proposed method for a variety of structural systems, and for different types of control parameters and imperfections such as geometric defects, load imperfections and thermal stresses. In all cases, the proposed method gives a wide range of stability boundaries with good precision and adequate computational efficiency.

\section{Acknowledgements}

The authors gratefully acknowledge the helpful suggestions received from the anonymous reviewers. The quality of this article has benefited substantially from their comments.

The first author also acknowledges the contributions Prof. Rezaiee Pajand had to his education.

\section{References}

[1] K. Ikeda, M. Ohsaki, Generalized sensitivity and probabilistic analysis of buckling loads of structures, International Journal of Non-Linear Mechanics 42 (5) (2007) 733-743.

[2] E. Parente Jr, J. B. M. de Sousa Jr, Design sensitivity analysis of nonlinear structures subjected to thermal loads, Computers \& Structures 86 (11-12) (2008) 1369-1384.

[3] K. Huseyin, Nonlinear theory of elastic stability, Noordhoff, 1975.

[4] B. W. R. Forde, S. F. Stiemer, Improved arc length orthogonality methods for nonlinear finite element analysis, Computers \& Structures 27 (5) (1987) 625-630. 
[5] E. Riks, An incremental approach to the solution of snapping and buckling problems, International Journal of Solids and Structures 15 (7) (1979) 529551.

[6] M. A. Crisfield, An arc-length method including line searches and accelerations, International Journal for Numerical Methods in Engineering 19 (9) (1983) 1269-1289.

[7] H. Chen, G. E. Blandford, Work-increment-control method for non-linear analysis, International Journal for Numerical Methods in Engineering 36 (6) (1993) 909-930.

[8] B. R. Widjaja, Path-following technique based on residual energy suppression for nonlinear finite element analysis, Computers \& Structures 66 (2-3) (1998) 201-209.

[9] M. Rezaiee-Pajand, M. Tatar, B. Moghaddasie, Some geometrical bases for incremental-iterative methods, International Journal of EngineeringTransactions B: Applications 22 (3) (2009) 245-256.

[10] P. Wriggers, W. Wagner, C. Miehe, A quadratically convergent procedure for the calculation of stability points in finite element analysis, Computer Methods in Applied Mechanics and Engineering 70 (3) (1988) 329-347.

[11] F. Fujii, K. Ikeda, H. Noguchi, S. Okazawa, Modified stiffness iteration to pinpoint multiple bifurcation points, Computer Methods in Applied Mechanics and Engineering 190 (18-19) (2001) 2499-2522.

[12] J. M. Battini, C. Pacoste, A. Eriksson, Improved minimal augmentation procedure for the direct computation of critical points, Computer Methods in Applied Mechanics and Engineering 192 (16-18) (2003) 2169-2185.

[13] P. Wriggers, J. C. Simo, A general procedure for the direct computation of turning and bifurcation points, International Journal for Numerical Methods in Engineering 30 (1) (1990) 155-176. 
[14] F. Fujii, S. Okazawa, Pinpointing bifurcation points and branch-switching, ASCE Journal of Engineering Mechanics 123 (3) (1997) 179-189.

[15] F. Fujii, E. Ramm, Computational bifurcation theory: path-tracing, pinpointing and path-switching, Engineering Structures 19 (5) (1997) 385-392.

[16] E. Parente Jr, A. S. de Holanda, S. M. B. A. da Silva, Tracing nonlinear equilibrium paths of structures subjected to thermal loading, Computational Mechanics 38 (6) (2006) 505-520.

[17] M. Ohsaki, K. Ikeda, Imperfection sensitivity of degenerate hilltop branching points, International Journal of Non-Linear Mechanics 44 (3) (2009) 324-336.

[18] M. Ohsaki, Design sensitivity analysis and optimization for nonlinear buckling of finite-dimensional elastic conservative structures, Computer Methods in Applied Mechanics and Engineering 194 (30-33) (2005) 3331-3358.

[19] J. M. T. Thompson, G. W. Hunt, A general theory of elastic stability, J. Wiley, 1973.

[20] B. Wu, Z. Wang, A perturbation method for the determination of the buckling strength of imperfection-sensitive structures, Computer Methods in Applied Mechanics and Engineering 145 (3-4) (1997) 203-215.

[21] L. A. Godoy, E. G. Banchio, Singular perturbations for sensitivity analysis in symmetric bifurcation buckling, International Journal for Numerical Methods in Engineering 52 (12) (2001) 1465-1485.

[22] R. Casciaro, G. Salerno, A. D. Lanzo, Finite element asymptotic analysis of slender elastic structures: a simple approach, International Journal for Numerical Methods in Engineering 35 (7) (1992) 1397-1426.

[23] R. Casciaro, G. Garcea, G. Attanasio, F. Giordano, Perturbation approach to elastic post-buckling analysis, Computers \& Structures 66 (5) (1998) $585-595$. 
[24] G. Garcea, A. Madeo, G. Zagari, R. Casciaro, Asymptotic post-buckling FEM analysis using corotational formulation, International Journal of Solids and Structures 46 (2) (2009) 377-397.

[25] B. Wu, Direct calculation of buckling strength of imperfect structures, International Journal of Solids and Structures 37 (11) (2000) 1561-1576.

[26] K. K. Choong, J. Y. Kim, A numerical strategy for computing the stability boundaries for multi-loading systems by using generalized inverse and continuation method, Engineering Structures 23 (6) (2001) 715-724.

[27] M. A. Crisfield, A fast incremental/iterative solution procedure that handles "snap-through", Computers \& Structures 13 (1981) 55-62.

[28] S. Krenk, An orthogonal residual procedure for non-linear finite element equations, International Journal for Numerical Methods in Engineering 38 (5) (1995) 823-839.

[29] M. A. Crisfield, Non-linear Finite Element Analysis of Solids and Structures, Volume 1: Essentials, J. Wiley and Sons, Chichester, 1991.

[30] E. Ramm, Strategies for tracing the nonlinear response near limit points, Springer, 1981.

[31] A. Magnusson, I. Svensson, Numerical treatment of complete loaddeflection curves, International Journal for Numerical Methods in Engineering 41 (5) (1998) 955-971.

[32] E. A. de Souza Neto, Y. T. Feng, On the determination of the path direction for arc-length methods in the presence of bifurcations and 'snap-backs', Computer Methods in Applied Mechanics and Engineering 179 (1-2) (1999) $81-89$.

[33] S. Lopez, Detection of bifurcation points along a curve traced by a continuation method, International Journal for Numerical Methods in Engineering 53 (4) (2002) 983-1004. 
[34] M. Rezaiee-Pajand, H. R. Vejdani-Noghreiyan, Computation of multiple bifurcation point, Engineering Computations 23 (5) (2006) 552-565.

[35] J. B. Hiriart-Urruty, D. Ye, Sensitivity analysis of all eigenvalues of a symmetric matrix, Numerische Mathematik 70 (1) (1995) 45-72.

[36] K. Ikeda, K. Murota, A. Yanagimoto, H. Noguchi, Improvement of the scaled corrector method for bifurcation analysis using symmetry-exploiting block-diagonalization, Computer Methods in Applied Mechanics and Engineering 196 (9-12) (2007) 1648-1661.

[37] E. Parente Jr., L. E. Vaz, On evaluation of shape sensitivities of non-linear critical loads, International Journal for Numerical Methods in Engineering 56 (6) (2003) 809-846.

[38] A. Eriksson, Fold lines for sensitivity analyses in structural instability, Computer Methods in Applied Mechanics and Engineering 114 (1-2) (1994) $77-101$.

[39] A. Eriksson, C. Pacoste, A. Zdunek, Numerical analysis of complex instability behaviour using incremental-iterative strategies, Computer Methods in Applied Mechanics and Engineering 179 (3) (1999) 265-305.

[40] M. Kleiber, T. D. Hien, Parameter sensitivity of inelastic buckling and post-buckling response, Computer Methods in Applied Mechanics and Engineering 145 (3) (1997) 239-262.

[41] M. Deml, W. Wunderlich, Direct evaluation of the 'worst' imperfection shape in shell buckling, Computer Methods in Applied Mechanics and Engineering 149 (1) (1997) 201-222.

[42] W. Wunderlich, U. Albertin, Analysis and load carrying behaviour of imperfection sensitive shells, International Journal for Numerical Methods in Engineering 47 (1-3) (2000) 255-273. 
[43] J. E. Dennis Jr., R. B. Schnabel, Numerical methods for unconstrained optimization and nonlinear equations, Vol. 16, Society for Industrial Mathematics, 1996.

[44] M. A. Crisfield, Non-linear Finite Element Analysis of Solids and Structures, Volume 2: Advanced Topics, J. Wiley and Sons, Chichester, 1997.

[45] Wolfram Mathematica $8.0 \quad$ Documentation Center, Wolfram Research Inc., Champaign, IL, USA, http://reference.wolfram.com/mathematica/guide/Mathematica.html.

[46] P. G. Bergan, Solution algorithms for nonlinear structural problems, Computers \& Structures 12 (4) (1980) 497-509.

[47] S. Krenk, Non-linear Modeling and Analysis of Solids and Structures, Cambridge University Press, 2009.

[48] K. Ikeda, M. Ohsaki, Y. Kanno, Imperfection sensitivity of hilltop branching points of systems with dihedral group symmetry, International Journal of Non-Linear Mechanics 40 (5) (2005) 755-774.

[49] I. Stanciulescu, T. Mitchell, Y. Chandra, T. Eason, M. Spottswood, A lower bound on snap-through instability of curved beams under thermomechanical loads, International Journal of Non-Linear Mechanics 47 (5) (2012) 561-575.

[50] A. Eriksson, Derivatives of tangential stiffness matrices for equilibrium path descriptions, International Journal for Numerical Methods in Engineering 32 (5) (1991) 1093-1113.

[51] C. Cho, H. C. Park, S. W. Lee, Stability analysis using a geometrically nonlinear assumed strain solid shell element model, Finite Elements in Analysis and Design 29 (2) (1998) 121-135.

[52] A. Eriksson, Equilibrium subsets for multi-parametric structural analysis, Computer Methods in Applied Mechanics and Engineering 140 (3) (1997) 305-327. 